\begin{tabular}{cc} 
Sharif University of Technology \\
Scientia Iranica \\
SCIENTIA & Transactions B: Mechanical Engineering \\
I RAN I CA & \\
\hline
\end{tabular}

\title{
Adaptive control of a cable-actuated parallel manipulator mounted on a platform with differential wheels under payload uncertainty
}

\author{
M.H. Korayem* and M. Yousefzadeh \\ Robotic Research Laboratory, Center of Excellence in Experimental Solid Mechanics and Dynamics, School of Mechanical \\ Engineering, Iran University of Science and Technology, Tehran, Iran. \\ Received 19 December 2017; received in revised form 17 July 2018; accepted 1 October 2018
}

\author{
KEYWORDS \\ Cable-actuated \\ Parallel Manipulators \\ (CPMs); \\ Mobile robots with \\ differential wheels; \\ Non-holonomic \\ constraints; \\ Adaptive control; \\ Sliding mode control.
}

\begin{abstract}
Cable-actuated Parallel Manipulators (CPMs) are widely employed for object handling applications. In order to displace the carried object along the ground to an unlimited distance, CPMs can be mounted on Wheeled Mobile Robots (WMRs). The derivation of the dynamic equations of motion for this integrated system is presented using the Lagrange method. Since the inertia of the moving load is the main source of uncertainty in the load-carrying task, an adaptive control approach is considered for the CPM, whereas the WMR uses the feedback linearized sliding mode approach. In order to maintain the end-effector of the CPM in its relative workspace in the WMR frame, the convergence rate in the two controllers should be similar. Decentralization of the control law can be accomplished if the inertia of the CPM motors is negligible compared with that of other systems. This assumption is shown to be applicable if an introduced index is small enough to have noticeable effect on the tracking error.

(C) 2020 Sharif University of Technology. All rights reserved.
\end{abstract}

\section{Introduction}

The larger workspace and higher ratio of carrying load to the manipulator's moving weight are the main characteristics of Cable-Actuated Parallel Manipulators (CPMs). However, carrying a heavy load with lengthy cables may lead to a considerable increase in the system flexibility, which may lead to the instability of a closedloop system [1]. Moreover, in some operation fields, the required horizontal workspace in comparison to the height changes of the end-effector is significant. Therefore, the cable robot can encounter high cable tensions. The lengthy cable issues and the need

\footnotetext{
*. Corresponding author.

E-mail addresses: hkorayem@iust.ac.ir (M.H. Korayem); ma.yousef@gmail.com (M. Yousefzadeh)
}

for large operation workspace can be addressed by mounting the CPM on a wheeled mobile platform. In this regard, the dynamic equations of a CPM hung from a helicopter were obtained by $\mathrm{Oh}$ et al. [2]. In this study, the helicopter was navigated using a slow-rate controller for the translational motion and a fast-rate controller for attitude control. The CPM was controlled using a typical sliding mode method. Korayem et al. [3] presented Feedback Linearization (FL) control of a 6-DOF under-constrained cablesuspended manipulator with a wheeled mobile platform. For the same robotic system, Korayem et al. [4] incorporated cable sagging in the calculation while the end-effector and the wheeled platform were controlled independently. In order to ensure a wide operation area and good mobility, $\mathrm{Hu}$ et al. [5] introduced an integrated design of a mobile parallel robot with threewheeled limbs. Each limb consists of the prismatic and universal joints, as well as omni-directional wheels at 
the end of the limb. The 500-m aperture spherical radio telescope (FAST) in China shows the application of carrying a parallel robot with a robotic system [6]. The FAST mechanism is a cable-actuated parallel robot carrying a Stewart manipulator, which compensates the wind-induced disturbances imposed on the receiver.

The uncertainties and nonlinear dynamics of the parallel manipulators are the main challenges in the controller design. Robust and adaptive controllers are the two major approaches to addressing uncertain nonlinear systems. In this regard, for the control of a singularity-free planar parallel robot, Sliding Mode Control (SMC) was employed to address the modeled uncertainties, whereas an observer was used to determine the external disturbances [7]. For the robot manipulator control, Asl et al. [8] proposed a nonsingular terminal SMC. In order to reduce the control signal chattering, the discontinuous term of the control signal was obtained using a neural network method. In this study, the feedback was obtained from the unscented Kalman filter and a modified evolutionary algorithm to estimate the covariance of the process and measurement noise. Yang et al. [9] employed the impedance control law for a 3-DOF cable robot aiding the human arm rehabilitation. They adjusted the controller gains by a fuzzy tuner considering the cable length and the change of cable length.

Generally, due to the estimation of the system's unknown parameters, adaptive controllers require a greater load of computation than robust controllers. On the other hand, robust controllers require the upper bound of the system uncertainties. In order to enable the robust controller to operate over the whole domain of possible uncertainties, the uncertainty bound is selected large enough. This can result in high switching gain and control effort. To alleviate this problem, some controllers are used to estimate the unknown parameters using adaptation laws and address the effects of external disturbance using robust control. Lee et al. [10] introduced a model reference adaptive sliding scheme for three-dimensional overhead crane control without priori information of the system parameters. Indeed, this approach still requires the bound of uncertainties. Sun and Ma [11] proposed an adaptive fractional-order terminal SMC for tracking control of linear motors. In this study, the adaptive term ensures precise finite time convergence of the sliding mode variable while addressing the bounded structured uncertainties. They also employed a sliding mode disturbance observer to reduce measurement noise and uncertainties including parametric uncertainty and disturbances.

Adaptive-robust controllers can apply adaptation laws to estimate the upper bound of the uncertainties so that the overestimation of the switching gain can be addressed [12]. For the SMC of a fully constrained cable-driven robot, an adaptation law based on the approach proposed by Babaghasabha et al. [13] to estimate the upper bound of uncertainties required to determine the control gain [14]. Robust adaptive control of the payload carried by an offshore shipmounted crane, subjected to unknown parameters and sea wave disturbances, was proposed by Qian et al. [15]. They employed adaptation laws to estimate the upper bound of the overall system uncertainties. Qi et al. [16] presented the SMC law of the 4-DOF parallel robot with a fuzzy-based adaptive boundary layer and switching gains. The controller was used in combination with another fuzzy adaptive controller to reduce the chattering by compensating the unknown dynamics. For the control of a cable robot, El-Ghazaly et al. [17] proposed an adaptive terminal SMC method to improve the robustness despite the end-effector inertial uncertainties. Schenk et al. [18] presented trajectory tracking of cable robots in the presence of model uncertainties and external disturbances by an adaptive super twisting controller, which adapts the gains. Compared to the controller proposed in the referenced study [17], this controller produces fewer tracking errors and less chattering in the control input. Torabi et al. [19] employed a combination of an adaptive SMC scheme and the admittance method for controlling an exoskeleton rehabilitation robot, interacting with the human lower limb. The adaptive gain rate was defined in proportion to the sliding variable. Moreover, by linearly parameterizing the robot dynamic model in terms of unknown parameters and obtaining the corresponding regressor matrix, the structured unknown parameters were estimated.

Since CPMs are used as a crane in many applications, the mass and moment of inertias of the payload are usually unknown. Therefore, in this paper, an adaptive control approach is considered for the CPM part of the robot. Similar closed-loop dynamics is required for the CPM and the mobile platform to prevent the possibility of losing the tension in some cables. Therefore, the controllers of the CPM and the mobile base are designed similarly to the performance of FL dynamics. In Section 2 of this paper, the equations of motion of the proposed robotic system are described. Section 3 deals with the control law of the combined system. Sections 4 and 5 present the verification of the approach based on simulation and experimental results.

\section{Equations of motion}

\subsection{System architecture}

The integrated system studied in this study is a CPM with six degrees of freedom carried by a differential Wheeled Mobile Robot (WMR) through six cable actuators. The schematic view of the two parts of 


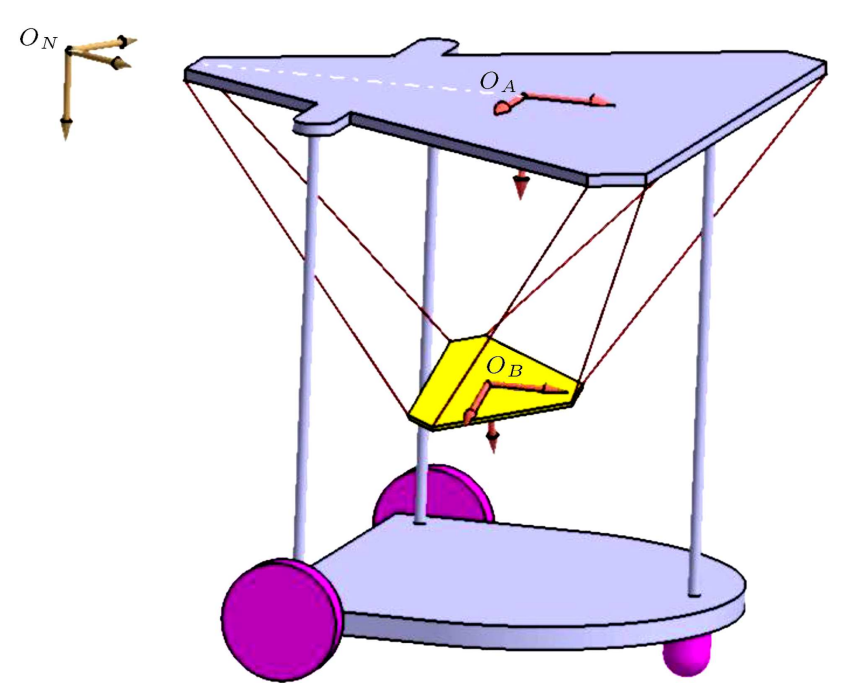

Figure 1. Schematic view of the wheeled mobile Cable-actuated Parallel Manipulators (CPM).

the system is shown in Figure 1. The wheeled mobile platform is driven by two fixed independent wheels. Since the CPM is of the under-constrained type, the motion of the system is considered without sudden change and high acceleration to maintain the cables in tension. As a result, the slippage of the wheels on the ground is not considered in this study.

\subsection{Kinematic equations}

The kinematic equations presented in this paper were derived from a study by Korayem et al. [3] in detail. However, for the sake of introducing the variables, this section presents the definition of the variables and kinematics of the system.

The subscripts $A, B$, and $N$ indicate the Cartesian system on the mobile base, the end-effector, and the ground, respectively. Due to the gentle trajectory tracking, the wheels do not slip. The mobile platform pose is determined by its mass center (C.G.) position, $\left[x_{A N}, y_{A N}\right]^{T}$, and orientation, $\varphi$. Considering the wheels angular velocity, $\dot{\boldsymbol{\theta}}=\left[\dot{\theta}_{r}, \dot{\theta}_{l}\right]^{T}$, and the mobile platform pose $\mathbf{x}_{\mathbf{A}}=\left[x_{A N}, y_{A N}, \mathbf{0}_{\mathbf{1} \times \mathbf{3}}, \varphi\right]^{T}$, the platform kinematics can be expressed as follows:

$$
\begin{aligned}
\dot{\mathbf{x}}_{\mathbf{A}} & =\mathbf{C}_{\mathbf{6}} \dot{\boldsymbol{\theta}} \\
\mathbf{C}_{\mathbf{6}} & =\frac{r_{w h}}{2 b}\left[\begin{array}{cc}
d \sin \varphi+b \cos \varphi & -d \sin \varphi+b \cos \varphi \\
-d \cos \varphi+b \sin \varphi & d \cos \varphi+b \sin \varphi \\
\mathbf{0}_{\mathbf{3} \times \mathbf{1}} & \mathbf{0}_{\mathbf{3} \times \mathbf{1}} \\
-1 & 1
\end{array}\right]
\end{aligned}
$$

where $r_{w h}, 2 b$, and $d$ represent the wheel radius, the axle track, and the distance from the platform mass center to its axle, respectively.

The end-effector pose consists of the position vector denoted by ${ }^{\mathbf{N}} \mathbf{x}_{\mathbf{B N}}$ and the Euler angles represented with $\boldsymbol{\Psi}_{\mathrm{BN}}$ :

$$
\mathrm{x}_{\mathrm{B}}=\left[\begin{array}{c}
\mathrm{N}_{\mathrm{x}_{\mathrm{BN}}} \\
\mathbf{\Psi}_{\mathrm{BN}}
\end{array}\right] .
$$

The inverse kinematics equation of the cable robot relates the cable motor rotation vector, $\boldsymbol{\beta}$, to the mobile base pose, $\mathbf{x}_{\mathbf{A}}$, and the CPM pose, $\mathbf{x}_{\mathbf{B}}$, as follows:

$$
\dot{\boldsymbol{\beta}}=\frac{1}{r} \boldsymbol{\Lambda}^{\mathbf{T} \mathbf{A}} \overline{\mathbf{R}}_{\mathbf{B}}\left(\mathbf{C}_{\mathbf{1}} \dot{\mathbf{x}}_{\mathbf{A}}+\mathbf{C}_{\mathbf{2}} \mathbf{C}_{\mathbf{8}} \dot{\mathbf{x}}_{\mathbf{B}}\right)
$$

where the scalar constant, $r$, is the cable drum radius. The expression of the coefficient matrices, $\mathbf{C}_{\mathbf{1}}$ and $\mathbf{C}_{\mathbf{2}}$, and the Jacobian matrix, $\boldsymbol{\Lambda}$, are provided in [3].

\subsection{Dynamic equations of the wheeled CPM}

The dynamic equations of motion can be obtained using the Lagrangian formulation. The kinetic energy of the system can be expressed as follows:

$$
\begin{aligned}
\mathrm{KE}= & \frac{1}{2} \dot{\mathbf{x}}_{\mathbf{A}}^{\mathbf{T}}\left[\mathbf{M}_{\mathbf{A}}\right] \dot{\mathbf{x}}_{\mathbf{A}}+\frac{1}{2}\left(\mathbf{C}_{\mathbf{8}} \dot{\mathbf{x}}_{\mathbf{B}}\right)^{\mathbf{T}}\left[\mathbf{M}_{\mathbf{B}}\right] \mathbf{C}_{\mathbf{8}} \dot{\mathbf{x}}_{\mathbf{B}} \\
& +\frac{1}{2} \dot{\boldsymbol{\theta}}^{\mathbf{T}} \mathbf{I}_{\mathbf{w}} \dot{\boldsymbol{\theta}}+\frac{1}{2}\left(\dot{\boldsymbol{\beta}}+\mathbf{C}_{\mathbf{1 0}} \dot{\mathbf{x}}_{\mathbf{A}}\right)^{\mathbf{T}} \mathbf{I}_{\mathbf{m}}\left(\dot{\boldsymbol{\beta}}+\mathbf{C}_{\mathbf{1 0}} \dot{\mathbf{x}}_{\mathbf{A}}\right) \\
\mathbf{C}_{\mathbf{1 0}}= & {\left[\mathbf{0}_{\mathbf{6} \times \mathbf{5}}, \mathbf{1}_{\mathbf{6} \times \mathbf{1}}\right] \quad\left[\mathbf{M}_{\mathbf{A}}\right]=\left[\begin{array}{cc}
m_{A} \mathbf{I}_{\mathbf{3}} & \mathbf{0}_{\mathbf{3}} \\
\mathbf{0}_{\mathbf{3}} & \mathbf{I}_{\mathbf{A}}
\end{array}\right] } \\
{\left[\mathbf{M}_{\mathbf{B}}\right]=} & {\left[\begin{array}{cc}
m_{B} \mathbf{I}_{\mathbf{3}} & \mathbf{0}_{\mathbf{3}} \\
\mathbf{0}_{\mathbf{3}} & \mathbf{I}_{\mathbf{B}}
\end{array}\right] }
\end{aligned}
$$

The mass matrices of the mobile base $\left[\mathbf{M}_{\mathbf{A}}\right]$ and the end-effector $\left[\mathbf{M}_{\mathbf{B}}\right]$ consist of the corresponding mass and inertia tensor, i.e., $\left(m_{A}, \mathbf{I}_{\mathbf{A}}\right)$ and $\left(m_{B}, \mathbf{I}_{\mathbf{B}}\right)$. The matrix $\mathbf{I}_{\mathbf{m}}$ represents the moment of inertia of the CPM motors obtained considering the cable drum rotation. The moment of inertia of the mobile base actuators is $\mathbf{I}_{\mathbf{w}}$. The generalized power of the system including the applied motor torques and the gravitation can be expressed as follows:

$$
\dot{W}=\boldsymbol{\tau}_{\boldsymbol{\beta}}^{\mathbf{T}} \dot{\boldsymbol{\beta}}+\boldsymbol{\tau}_{\boldsymbol{\theta}}^{\mathbf{T}} \dot{\boldsymbol{\theta}}+\left[\mathbf{M}_{\mathbf{B}}\right] \mathbf{g}\left[\begin{array}{lll}
\mathbf{0}_{\mathbf{1} \times \mathbf{2}} & \dot{z}_{B N} & \mathbf{0}_{\mathbf{1} \times \mathbf{3}}
\end{array}\right]^{\mathrm{T}},
$$

where $\boldsymbol{\tau}_{\boldsymbol{\beta}}=\left[\tau_{1}, \cdots, \tau_{6}\right]^{T}$ is the cable motor torque vector, $\boldsymbol{\tau}_{\boldsymbol{\theta}}=\left[\tau_{r}, \tau_{l}\right]^{T}$ is the wheel torque vector, and $z_{B N}$ is the end-effector vertical position in the reference frame. The dynamic equation of the combined system is obtained by substituting Eqs. (4) and (5) and the kinematic equations in the general form of the EulerLagrange formulation and eliminating the Lagrange multipliers. After conducting some mathematical operations, the combined system dynamics can be represented as follows:

$$
\mathbf{M}\left[\begin{array}{c}
\ddot{\mathrm{x}}_{\mathrm{B}} \\
\ddot{\theta}
\end{array}\right]+\mathbf{C}+\mathbf{G}=\mathbf{F}\left[\begin{array}{c}
\tau_{\beta} \\
\tau_{\theta}
\end{array}\right],
$$

where, the matrices $\mathbf{M}, \mathbf{C}$, and $\mathbf{G}$ are defined as shown in Box I. 


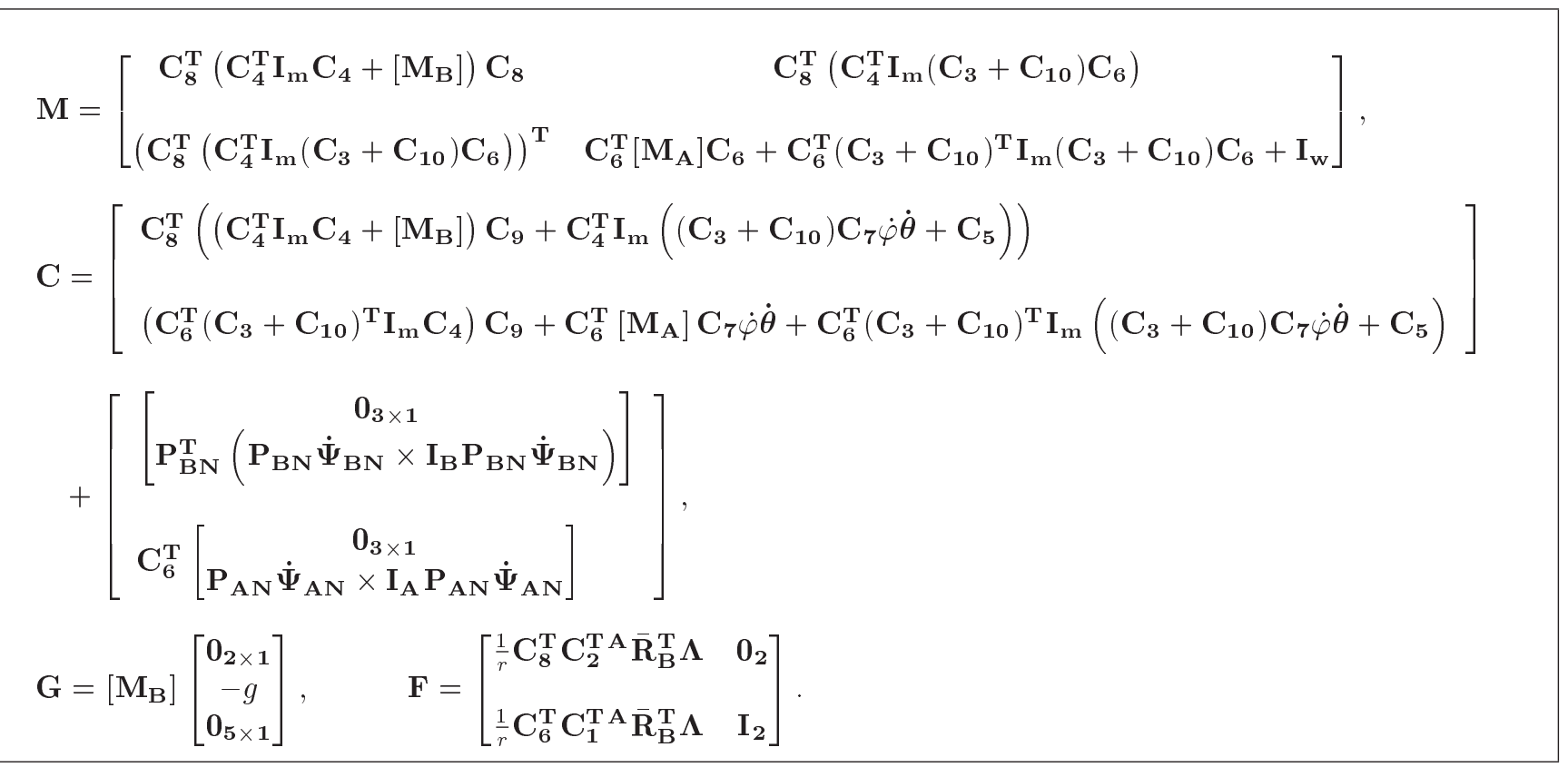

According to Eq. (6), the main source of the dynamic coupling between the mobile base and the CPM is produced by the terms having $\mathbf{I}_{\mathbf{m}}$ and the term $\frac{1}{r} \mathbf{C}_{\mathbf{6}}^{\mathbf{T}} \mathbf{C}_{\mathbf{1}}^{\mathbf{T} \mathbf{A}} \overline{\mathbf{R}}_{\mathbf{B}}^{\mathbf{T}} \boldsymbol{\Lambda}$ of the torque coefficient matrix, $\mathbf{F}$. Moreover, the term $\frac{1}{r} \mathbf{C}_{\mathbf{6}}^{\mathbf{T}} \mathbf{C}_{\mathbf{1}}^{\mathbf{T} \mathbf{A}} \overline{\mathbf{R}}_{\mathbf{B}}^{\mathbf{T}} \boldsymbol{\Lambda}$ in the torque coefficient matrix is related to the mobile base pose, showing the coupling between the CPM motor torques and the mobile base torques. However, given that the dynamic effect of the cable motor inertia, $\mathbf{I}_{\mathbf{m}} / r^{2}$, is negligible compared to the mass of the two parts of the system, the dynamic coupling in the control laws can be treated independently.

\section{Controller design}

Due to a wide range of payload weights and geometries in operation of cranes, the main source of uncertainty is considered to be the unknown payload inertia. Therefore, employing an adaptive law for the end-effector control would be a suitable choice. The end-effector should remain in the allowable space determined by the mobile base frame. Consequently, the two parts of the system should track the determined path with almost similar error dynamics. Therefore, to control the mobile, a robust FL approach with the same error dynamics as the end-effector controller is employed. When the mobile base is off the defined sliding surface, an auxiliary control signal pushes the mobile base toward the sliding surface. Once on the sliding surface, the error dynamics resembles the FL method to a greater degree.

\subsection{End-effector control}

In this paper, it is assumed that, in comparison with the inertias of the two parts, the moment of inertia of the CPM motors has a negligible effect on the system dynamics. Therefore, the terms including the parameter $\mathbf{I}_{\mathbf{m}}$ in Eq. (6) are ignored in the controller design. This assumption makes the effect of the mobile base motion on the end-effector dynamic negligible:

$$
\begin{aligned}
& \mathbf{M}_{\mathrm{e}} \ddot{\mathbf{x}}_{\mathrm{B}}+\mathrm{C}_{\mathrm{e}} \dot{\mathrm{x}}_{\mathrm{B}}+\mathrm{G}_{\mathrm{e}}=\mathrm{C}_{8}{ }^{\mathbf{T}} \mathbf{C}_{4}{ }^{\mathrm{T}} \tau_{\beta}, \\
& \mathbf{M}_{\mathbf{e}}=\mathbf{C}_{8}{ }^{\mathrm{T}}\left[\mathbf{M}_{\mathrm{B}}\right] \mathbf{C}_{8}, \\
& \mathrm{C}_{\mathbf{e}}=\mathrm{C}_{8}{ }^{\mathrm{T}}\left(\left[\mathrm{M}_{\mathrm{B}}\right] \dot{\mathbf{C}}_{8}+\left[\begin{array}{cc}
\mathbf{0}_{3} & \mathbf{0}_{3} \\
\mathbf{0}_{3} & \omega_{\mathrm{B}} \times \mathbf{I}_{\mathrm{B}} \mathbf{P}_{\mathrm{BN}}
\end{array}\right]\right), \\
& \mathbf{G}_{\mathbf{e}}=\mathbf{G}_{1: 6}
\end{aligned}
$$

where $\mathbf{M}_{\mathbf{e}}$ is the positive definite matrix, and $\dot{\mathbf{M}}_{\mathbf{e}}-2 \mathbf{C}_{\mathbf{e}}$ is a skew-symmetric matrix. A sliding vector is defined as follows:

$$
\mathbf{s}=\dot{\tilde{\mathbf{x}}}_{\mathbf{B}}+\lambda \tilde{\mathbf{x}}_{\mathbf{B}}, \quad \tilde{\mathbf{x}}_{\mathbf{B}}=\mathbf{x}_{\mathbf{B}_{\mathbf{d}}}-\mathbf{x}_{\mathbf{B}}
$$

where $\lambda$ is a positive value. In addition, the vector of parameter estimation error is defined as follows:

$$
\tilde{\mathbf{a}}=\hat{\mathbf{a}}-\mathbf{a},
$$

where $\mathbf{a}=\left[\begin{array}{llll}m_{b} & I_{B_{x}} & I_{B_{y}} & I_{B_{z}}\end{array}\right]^{T}$ denotes a series of exact inertia parameters, and $\hat{\mathbf{a}}$ indicates the estimation of a. A Lyapunov candidate is considered as follows:

$$
v=\frac{1}{2}\left(\mathbf{s}^{T} \mathbf{M}_{\mathbf{e}} \mathbf{s}+\tilde{\mathbf{a}}^{T} \Gamma^{-1} \tilde{\mathbf{a}}\right),
$$


where $\Gamma$ is a positive definite value used for tuning the parameter estimation speed. Differentiating Eq. (10) yields:

$$
\begin{aligned}
\dot{v}= & \mathbf{s}^{T}\left(\mathbf{M}_{\mathbf{e}}\left(\ddot{\mathbf{x}}_{\mathbf{B}_{\mathbf{d}}}+\lambda \dot{\tilde{\mathbf{x}}}_{\mathbf{B}}\right)+\mathbf{C}_{\mathbf{e}}\left(\dot{\mathbf{x}}_{\mathbf{B}_{\mathbf{d}}}+\lambda \tilde{\mathbf{x}}_{\mathbf{B}}\right)\right. \\
& \left.+\mathbf{G}_{\mathbf{e}}-\mathbf{C}_{\mathbf{8}}{ }^{T} \mathbf{C}_{\mathbf{4}}{ }^{T} \boldsymbol{\tau}_{\boldsymbol{\beta}}\right)+\dot{\hat{\mathbf{a}}}^{T} \Gamma^{-1} \tilde{\mathbf{a}} .
\end{aligned}
$$

The control law is considered as:

$$
\begin{aligned}
\boldsymbol{\tau}_{\boldsymbol{\beta}}= & \left(\mathbf{C}_{\mathbf{4}} \mathbf{C}_{\mathbf{8}}\right)^{-T}\left(\hat{\mathbf{M}}_{\mathbf{e}}\left(\ddot{\mathbf{x}}_{\mathbf{B}_{\mathbf{d}}}+\lambda \dot{\tilde{\mathbf{x}}}_{\mathbf{B}}+\lambda \mathbf{s}\right)\right. \\
& \left.+\hat{\mathbf{C}}_{\mathbf{e}}\left(\dot{\mathbf{x}}_{\mathbf{B}_{\mathbf{d}}}+\lambda \tilde{\mathbf{x}}_{\mathbf{B}}\right)+\hat{\mathbf{G}}_{\mathbf{e}}\right)=\left(\mathbf{C}_{\mathbf{4}} \mathbf{C}_{\mathbf{8}}\right)^{-T}(\mathbf{Y} \hat{\mathbf{a}}),
\end{aligned}
$$

where the symbol ( $)$ indicates that the associated matrix is obtained based on the estimated parameters. Substituting Eq. (12) into Eq. (11) gives the following:

$$
\dot{v}=-\mathbf{s}^{T} \lambda \mathbf{M}_{\mathbf{e}} \mathbf{s}+\dot{\hat{\mathbf{a}}}^{T} \Gamma^{-1} \tilde{\mathbf{a}}-\mathbf{s}^{T} \mathbf{Y} \tilde{\mathbf{a}} .
$$

By updating the values of the uncertain parameters:

$$
\dot{\hat{\mathbf{a}}}=-\Gamma \mathbf{Y}^{T} \mathbf{s},
$$

the derivative of Lyapunov function becomes:

$$
\dot{v}=-\mathbf{s}^{T} \lambda \mathbf{M}_{\mathbf{e}} \mathbf{s} \leq 0 .
$$

Eq. (15) is obtained regardless of the dynamic terms such as $\mathbf{I}_{\mathbf{m}}$. The expanded form of the control law in Eq. (12) as:

$$
\begin{aligned}
\boldsymbol{\tau}_{\boldsymbol{\beta}}= & \left(\mathbf{C}_{\mathbf{4}} \mathbf{C}_{\mathbf{8}}\right)^{-T}\left(\hat{\mathbf{M}}_{\mathbf{e}}\left(\ddot{\mathbf{x}}_{\mathbf{B}_{\mathbf{d}}}+2 \lambda \dot{\tilde{\mathbf{x}}}_{\mathbf{B}}+\lambda^{2} \tilde{\mathbf{x}}_{\mathbf{B}}\right)\right. \\
& \left.+\hat{\mathbf{C}}_{\mathbf{e}}\left(\dot{\mathbf{x}}_{\mathbf{B}_{\mathbf{d}}}+\lambda \tilde{\mathbf{x}}_{\mathbf{B}}\right)+\hat{\mathbf{G}}_{\mathbf{e}}\right),
\end{aligned}
$$

demonstrates a control approach similar to the FL method, except for a minor difference in the second term. However, although this control signal does not exactly cancel the nonlinear terms, it still guarantees exponential tracking convergence at the same rate as an exact cancellation.

\subsection{Wheeled mobile platform control}

By considering the state vector:

$$
\mathbf{x}=\left[\begin{array}{llll}
x_{A N} & y_{A N} & \boldsymbol{\theta}^{\mathbf{T}} & \dot{\boldsymbol{\theta}}^{\mathbf{T}}
\end{array}\right]^{T},
$$

the dynamic equations of motion can be expressed as follows:

$$
\dot{\mathrm{x}}=\left[\begin{array}{c}
\mathbf{S} \dot{\theta} \\
-\mathbf{M}_{\mathrm{p}}^{-1} \mathbf{C}_{\mathrm{p}}
\end{array}\right]+\left[\begin{array}{cc}
\mathbf{0}_{4 \times 6} & \mathbf{0}_{4 \times 2} \\
\mathbf{M}_{\mathrm{p}}^{-1} \mathbf{C}_{6}^{\mathbf{T}} \mathbf{C}_{3}^{\mathbf{T}} & \mathbf{M}_{\mathrm{p}}^{-1}
\end{array}\right]\left[\begin{array}{c}
\tau_{\beta} \\
\tau_{\theta}
\end{array}\right]
$$

where:

$$
\begin{aligned}
& \mathbf{S}=\left[\begin{array}{c}
\mathbf{C}_{\mathbf{6}_{(1: 2,1: 2)}} \\
\mathbf{I}_{2}
\end{array}\right], \quad \mathbf{M}_{\mathbf{p}}=\mathbf{C}_{\mathbf{6}}^{\mathbf{T}}\left[\mathbf{M}_{\mathbf{A}}\right] \mathbf{C}_{6}+\mathbf{I}_{\mathrm{w}} \\
& \mathrm{C}_{\mathrm{p}}=\mathrm{C}_{6}^{\mathbf{T}}\left[\mathrm{M}_{\mathbf{A}}\right] \mathbf{C}_{\mathbf{7}} \dot{\varphi} \dot{\boldsymbol{\theta}} \\
& +\mathbf{C}_{6}^{\mathbf{T}}\left[\begin{array}{c}
\mathbf{0}_{3 \times 1} \\
\mathbf{P}_{\mathrm{AN}} \dot{\Psi}_{\mathrm{AN}} \times \mathbf{I}_{\mathrm{A}} \mathbf{P}_{\mathrm{AN}} \dot{\Psi}_{\mathrm{AN}}
\end{array}\right]
\end{aligned}
$$

According to [20], the stability of the Feedback linearized system can be ensured by nonzero distance $d$ and positive velocity of the axle center. The mobile base position, $\mathbf{y}=\left(x_{A N}, y_{A N}\right)$, converges to the desired trajectory, $\mathbf{y}_{\text {des. }}$, using the control input as follows:

$$
\begin{gathered}
\left.\tau_{\theta_{\mathrm{FL}}}=\mathrm{M}_{\mathrm{p}}\left(\mathrm{S}_{1: 2 \times 1: 2}^{-1} v-\dot{\mathrm{S}}_{1: 2,1: 2} \dot{\theta}\right)\right)+\mathrm{C}_{\mathrm{p}} \\
-\mathrm{C}_{6}^{\mathrm{T}} \mathrm{C}_{3}^{\mathrm{T}} \tau_{\beta}, \\
\mathrm{e}=\mathrm{y}_{\mathrm{d}}-\mathrm{y}, \quad v=\ddot{\mathrm{y}}_{\mathrm{d}}+\mathrm{K}_{\mathrm{p}} \mathrm{e}+\mathrm{K}_{\mathrm{d}} \dot{\mathrm{e}},
\end{gathered}
$$

where the control gains, $\mathbf{K}_{\mathbf{p}}$ and $\mathbf{K}_{\mathbf{d}}$, are positive definite. By considering the control input in Eq. (18), the error dynamic of the mobile base is as follows:

$$
\ddot{\mathbf{e}}+\mathbf{K}_{\mathrm{d}} \dot{\mathrm{e}}+\mathbf{K}_{\mathbf{p}} \mathbf{e}=\mathbf{0} .
$$

By considering Eq. (16), $\mathbf{K}_{\mathbf{p}}=\lambda^{2} \mathbf{I}_{\mathbf{6}}$, and $\mathbf{K}_{\mathbf{d}}=2 \lambda \mathbf{I}_{\mathbf{6}}$, the mobile base error dynamic (Eq. (19)) can be made similar to the end-effector error dynamic. By defining a sliding surface as:

$$
\mathbf{s}=\dot{\mathbf{e}}+\mathbf{K}_{\mathrm{d}} \mathbf{e}+\mathbf{K}_{\mathbf{p}} \int \mathbf{e},
$$

the time rate of the Lyapunov candidate $v=\frac{1}{2} \mathbf{s}^{T} \mathbf{s}$ is given as follows:

$$
\begin{aligned}
\dot{v}= & \mathbf{s}^{T}\left(v-\dot{\mathbf{S}}_{\mathbf{1}: \mathbf{2}, \mathbf{1}: \mathbf{2}} \dot{\boldsymbol{\theta}}-\mathbf{S}_{\mathbf{1}: \mathbf{2} \times \mathbf{1 : 2}} \mathbf{M}_{\mathbf{p}}^{-\mathbf{1}}\left(-\mathbf{C}_{\mathbf{p}}\right.\right. \\
& \left.\left.+\mathbf{C}_{\mathbf{6}}^{\mathbf{T}} \mathbf{C}_{\mathbf{3}}^{\mathbf{T}} \tau_{\beta}+\tau_{\boldsymbol{\theta}}\right)\right) .
\end{aligned}
$$

By taking the control law of the mobile platform as:

$$
\tau_{\theta}=\tau_{\theta_{F L}}+\mathbf{M}_{\mathrm{p}} \mathbf{S}_{1: 2 \times 1: 2}{ }^{-1} \lambda \mathbf{M}_{\mathbf{e}_{2,1: 2}} \mathrm{~s},
$$

Eq. (21) can be expressed as:

$$
\dot{v}=-\mathbf{s}^{T} \lambda \mathbf{M}_{\mathbf{e}_{1: 2,1: 2}} \mathbf{s} \leq 0 .
$$

Based on the comparison of Eqs. (15) and (23), the convergence rate of the two controllers is similar. Therefore, if the end-effector is in the admissible workspace at the initial point, it will remain there during the trajectory tracking of the combined system. In the case that a wrench disturbance is applied to the WMR, an equivalent torque vector, $\boldsymbol{\tau}_{\mathbf{d}}$, can be considered to be exerted on the wheels. The wheels' 
disturbance is assumed to be bound by a positive value as $\left|\boldsymbol{\tau}_{\mathbf{d}}\right| \leq \tau_{m}\left[\begin{array}{ll}1 & 1\end{array}\right]^{T}$. By adding the discontinuous Sign function to the control law as:

$$
\begin{aligned}
\tau_{\boldsymbol{\theta}}= & \tau_{\theta_{F L}}+\mathbf{M}_{\mathrm{p}} \mathbf{S}_{1: 2 \times 1: 2}{ }^{-1} \lambda \mathbf{M}_{\mathbf{e}_{1: 2,1: 2}} \mathbf{s} \\
& +\mathbf{M}_{\mathrm{p}} \mathbf{S}_{1: 2 \times 1: 2}{ }^{-1} k \operatorname{Sign}(\mathbf{s}) .
\end{aligned}
$$

In Eq. (22), the derivative of the Lyapunov function becomes:

$$
\begin{aligned}
\dot{v}= & -\mathbf{s}^{T}\left(\lambda \mathbf{M}_{\mathbf{e}_{: 2,1: 2}} \mathbf{s}+k \operatorname{Sign}(\mathbf{s})\right. \\
& \left.+\mathbf{S}_{\mathbf{1 : 2 \times 1 : 2}} \mathbf{M}_{\mathbf{p}}{ }^{-1}\left(\boldsymbol{\tau}_{\mathbf{d}}\right)\right) \leq-\lambda m_{b}\|\mathbf{s}\|_{\mathbf{F}}^{2}-k\|\mathbf{s}\|_{\mathbf{F}} \\
& +\sqrt{2}\left\|\mathbf{S}_{\mathbf{1}: \mathbf{2} \times \mathbf{1}: 2}\right\|_{\mathbf{F}}\left\|\mathbf{M}_{\mathbf{p}}{ }^{-1}\right\|_{\mathbf{F}} \tau_{m}\|\mathbf{s}\|_{\mathbf{F}}
\end{aligned}
$$

where the Frobenius norms are given as:

$$
\begin{aligned}
& \left\|\mathbf{S}_{\mathbf{1 : 2 \times 1 : 2}}\right\|_{\mathbf{F}}=\sqrt{\frac{r_{w h}}{b}\left(b^{2}+d^{2}\right)}, \\
& \left\|\mathbf{M}_{\mathbf{p}}{ }^{-1}\right\|_{\mathbf{F}} \\
& =\frac{\sqrt{2}}{\left(m_{a} r_{w h}^{2}+2 I_{w}\right)\left(2 I_{w} b^{2}+I_{a z} r_{w h}^{2}+m_{a} d^{2} r_{w h}^{2}\right)} \\
& \quad\left(r_{w h}^{4}\left(m_{a}\left(d^{2}-b^{2}\right)+I_{a z}\right)^{2}\right. \\
& \left.+\left(m_{a} r_{w h}^{2}\left(b^{2}+d^{2}\right)+4 I_{w} b^{2}+I_{a z} r_{w h}^{2}\right)^{2}\right)^{\frac{1}{2}} .
\end{aligned}
$$

Therefore, the switching function gain can be obtained by:

$$
\begin{aligned}
k>\frac{2 \tau_{m} \sqrt{\frac{r_{w h}}{b}\left(b^{2}+d^{2}\right)}}{\left(m_{a} r_{w h}^{2}+2 I_{w}\right)\left(2 I_{w} b^{2}+I_{a z} r_{w h}^{2}+m_{a} d^{2} r_{w h}^{2}\right)} \\
\\
\left(r_{w h}^{4}\left(m_{a}\left(d^{2}-b^{2}\right)+I_{a z}\right)^{2}+\right. \\
\left.\quad\left(m_{a} r_{w h}^{2}\left(b^{2}+d^{2}\right)+4 I_{w} b^{2}+I_{a z} r_{w h}^{2}\right)^{2}\right)^{\frac{1}{2}} .
\end{aligned}
$$

In practice, the Sign function is replaced by $\tanh (\alpha s)$, where " $\alpha$ " denotes a positive scalar for scaling the boundary layer.

\section{Simulation}

The parameters of the robot are presented in [3]. The desired path of the mobile base and the CPM are considered as follows:

$$
\mathbf{x}_{\mathbf{A}}=\left[\begin{array}{c}
R \sin (\varphi) \\
-R \cos (\varphi) \\
0 \\
0 \\
0 \\
\varphi
\end{array}\right]
$$

$$
\begin{gathered}
\mathbf{x}_{\mathbf{B}}=\left[\begin{array}{c}
R \sin (\varphi) \\
R(-\cos (\varphi)+0.05 \sin (\varphi)) \\
0.8+0.1 \cos \left(\frac{8 \pi}{T} t\right) \\
0 \\
0 \\
\varphi
\end{array}\right], \\
\varphi=-\frac{4 \omega}{3 T^{2}}\left(t-\frac{T}{2}\right)^{3}+\omega t-\frac{\pi}{2} \quad \mathrm{rad},
\end{gathered}
$$

where:

$$
R=1.5 \mathrm{~m} \quad T=50 \mathrm{sec} \quad \omega=\frac{3 \pi}{T} \mathrm{rad} / \mathrm{sec} .
$$

The initial pose errors of the mobile base and the CPM are as follows:

$$
\begin{aligned}
& \mathbf{e}_{\mathbf{A}}=\left[\begin{array}{ll}
0.5 & -0.5
\end{array}\right]^{T}, \\
& \mathbf{e}_{\mathbf{B}}=\left[\begin{array}{llllll}
0.5 & -0.5 & -0.2 & -0.4 & -0.1 & -0.1
\end{array}\right]^{T} .
\end{aligned}
$$

The control parameters are selected as follows:

$$
\lambda=2 \mathbf{I}_{\mathbf{8}}, \quad \Gamma=1 .
$$

The tracking control effectiveness in addressing the uncertainty is investigated by assuming different masses of the end-effector and the mobile base platform in the controller and the plant presented as follows:

$$
m_{B_{C}}=m_{B_{P}}-10, \quad m_{A_{C}}=m_{A_{P}}-150,
$$

where the subscripts " $C$ " and " $P$ " denote the controller and the plant parameters. Figure 2 shows the control voltage of the mobile platform wheel motors. Since the applied voltages are limited to $\pm 12 \mathrm{v}$, the torques and motor speeds do not experience a further increase in the initial motion. The required high initial voltages are caused by the existing large errors at the start of the tracking. As shown in Figure 3, this overshoot can also be observed in the wheel motor speed. The motor speed at the end of the trajectory is zero, as expected from the

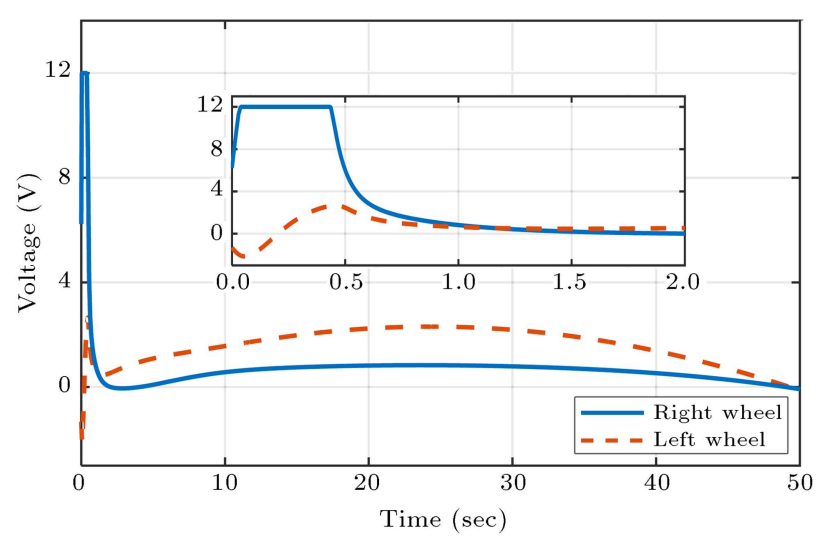

Figure 2. Voltage of wheel motors. 


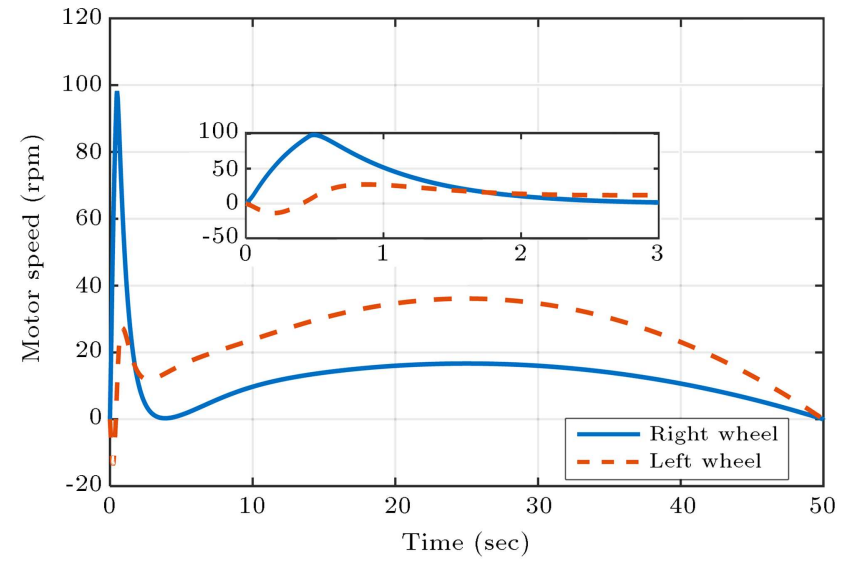

Figure 3. Wheel angular velocities.

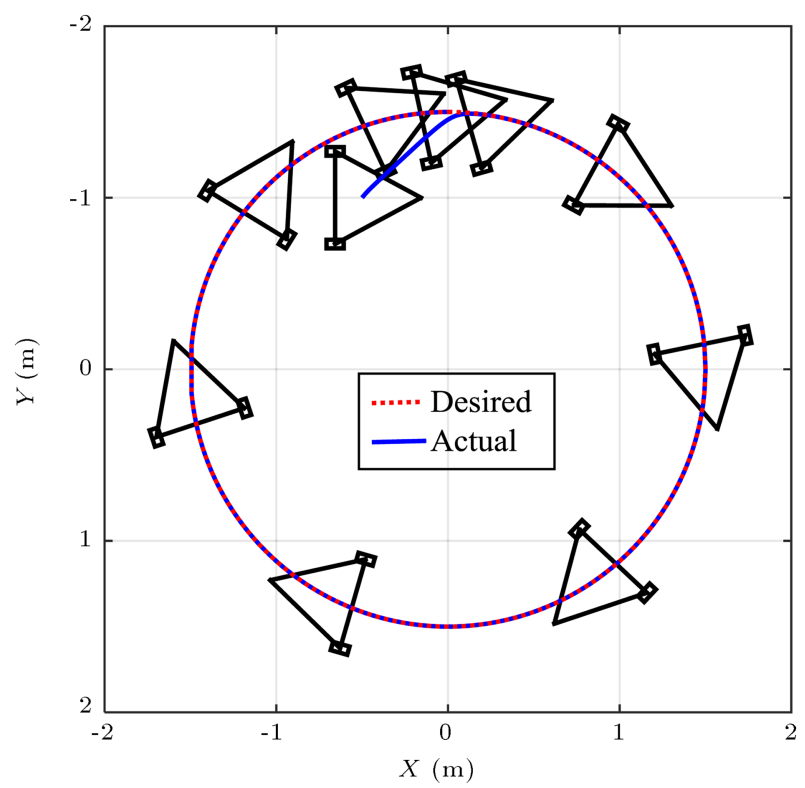

Figure 4. Platform trajectory.

desired trajectory formulation. Figure 4 demonstrates the trajectory tracking of the mobile base. Since the mobile base is initially positioned on the right side of the desired path, the initial right wheel speed is larger than the left wheel speed to force the mobile base to approach the desired trajectory. However, after reaching the desired trajectory, due to being positioned inside the circular path, the left wheel rotates with a higher speed. Figure 5 shows that the mobile base position error decreases in $6.4 \mathrm{sec}$, from $707 \mathrm{~mm}$ to the settling band of $0.05 \mathrm{~mm}$.

The control voltage of the CPM motors is shown in Figures 6 and 7 . The periodic fluctuations of the control input, caused by the vertical end-effector movement, can also be seen in the CPM motor speed in Figures 8 and 9. Figure 10 shows that the endeffector mass used in the control input calculation is updated from the initial guess of $1.1 \mathrm{~kg}$ to the actual mass of the end-effector, $11.1 \mathrm{~kg}$. The initial high

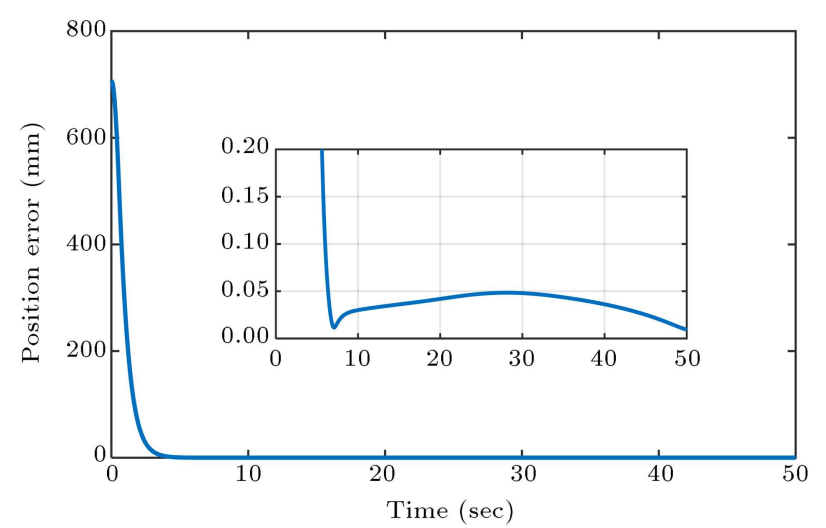

Figure 5. Platform position error.

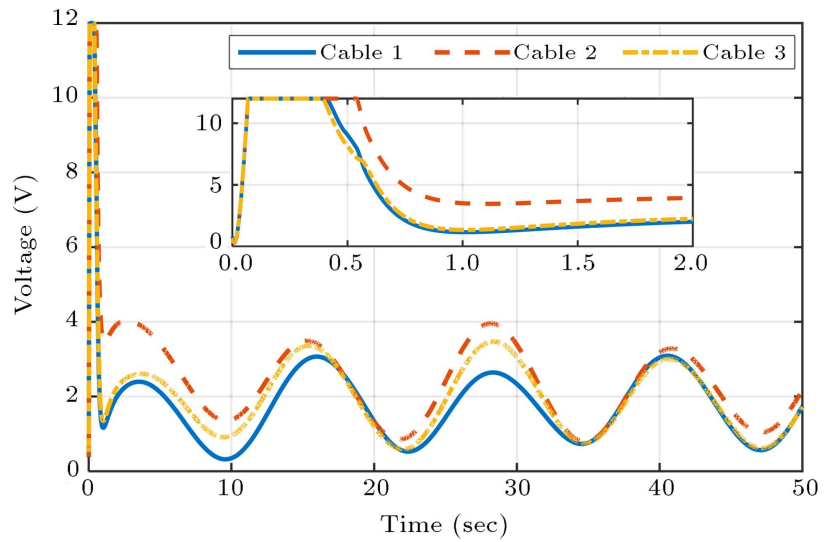

Figure 6. Voltages of the 1st, 2nd, and 3rd cable motors.

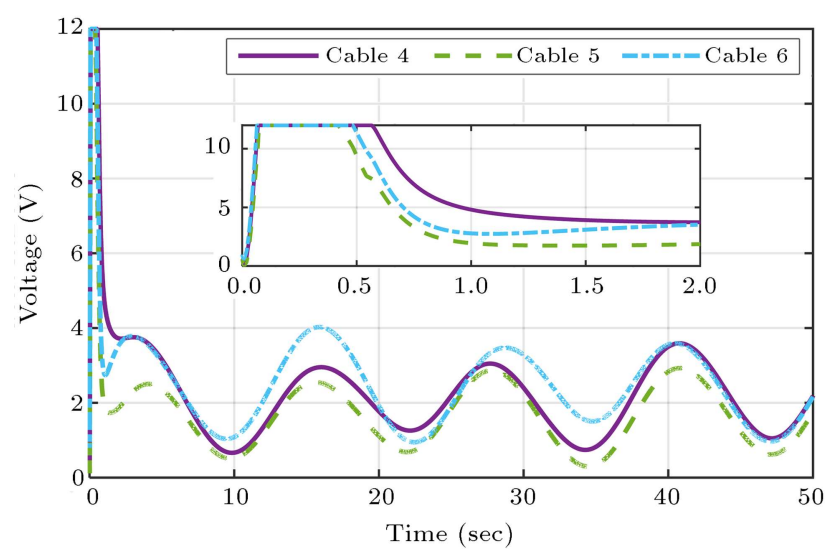

Figure 7. Voltages of the 4th, 5th, and 6th cable motors.

estimation error is caused by the saturation of the control input. However, after this short period, the estimation trend improves and converges to the actual value. The trajectory tracking of the CPM part of the system in the Cartesian space is demonstrated in Figure 11. Figure 12 shows that the 2-norm of the translational errors enters the settling band of $0.05 \mathrm{~mm}$ in $11.1 \mathrm{sec}$, from the initial value of $735 \mathrm{~mm}$. Moreover, Figure 13 shows that the end-effector orientation error, calculated based on the norm of the Euler angle errors, 


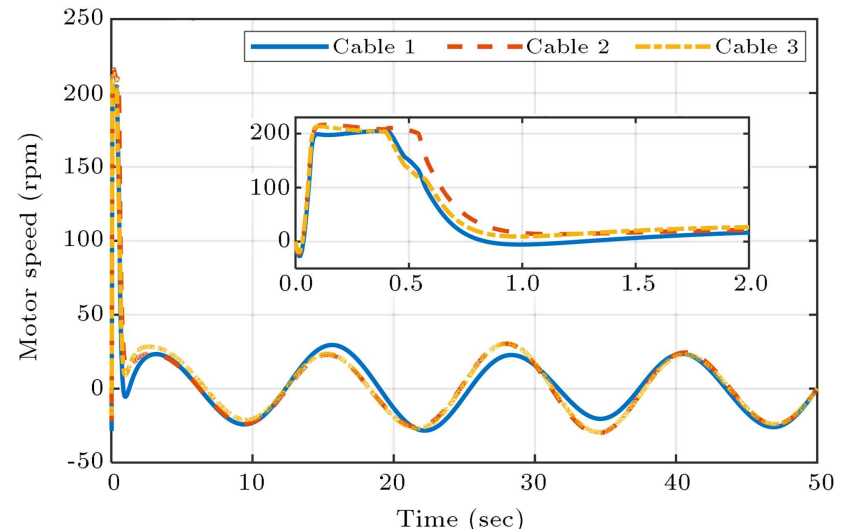

Figure 8. Angular velocities of the 1st, 2nd, and 3rd cable motors.

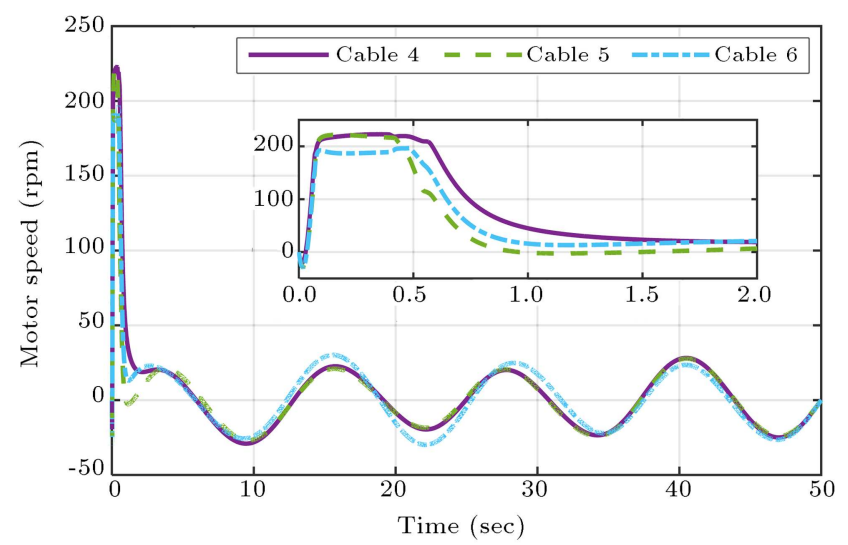

Figure 9. Angular velocities of the $4 \mathrm{th}, 5 \mathrm{th}$, and $6 \mathrm{th}$ cable motors.

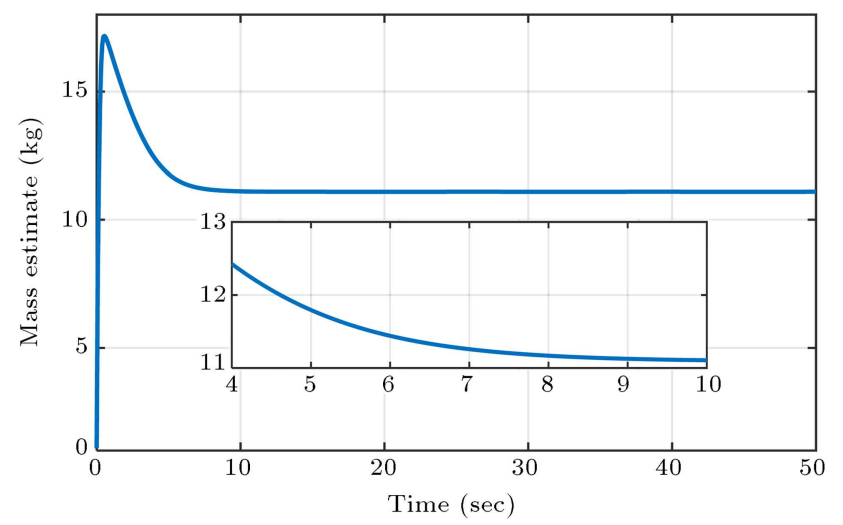

Figure 10. End-effector mass estimate.

decreases from $24.3 \mathrm{deg}$ to below $0.25 \mathrm{deg}$ in less than $5 \mathrm{sec}$.

Now, let's assume that the mobile base and the CPM are stationary at the following location:

$$
\mathbf{x}_{\mathbf{A}}=\mathbf{0}, \quad \mathbf{x}_{\mathbf{B}}=\left[\begin{array}{lll}
\mathbf{0}_{\mathbf{1} \times \mathbf{2}} & 0.8 & \mathbf{0}_{\mathbf{1} \times \mathbf{3}}
\end{array}\right]^{T} .
$$

For investigating the effect of the CPM motors' in ertia compared with the end-effector mass, the ratio of $\mathbf{I}_{\mathbf{m}}$

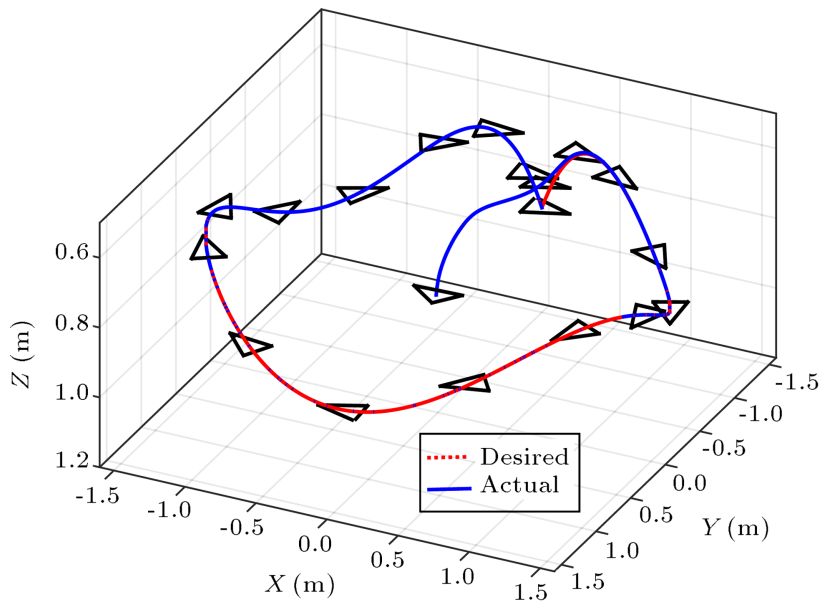

Figure 11. End-effector trajectory.

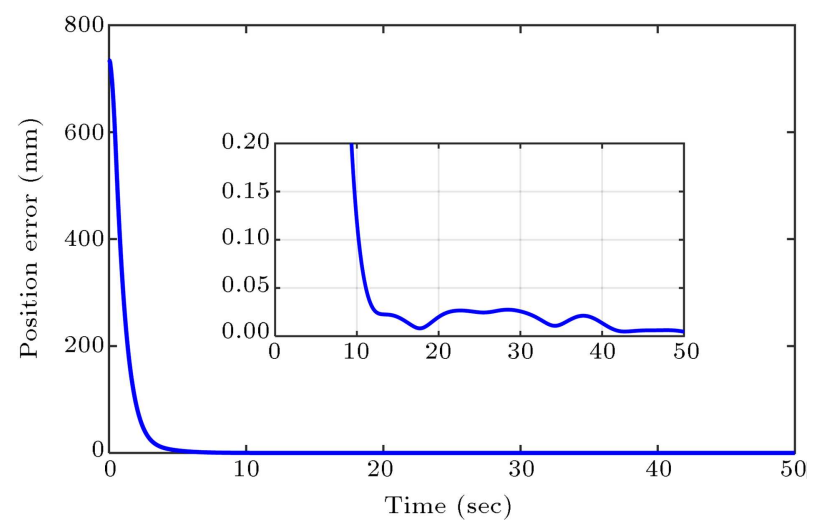

Figure 12. End-effector position error.

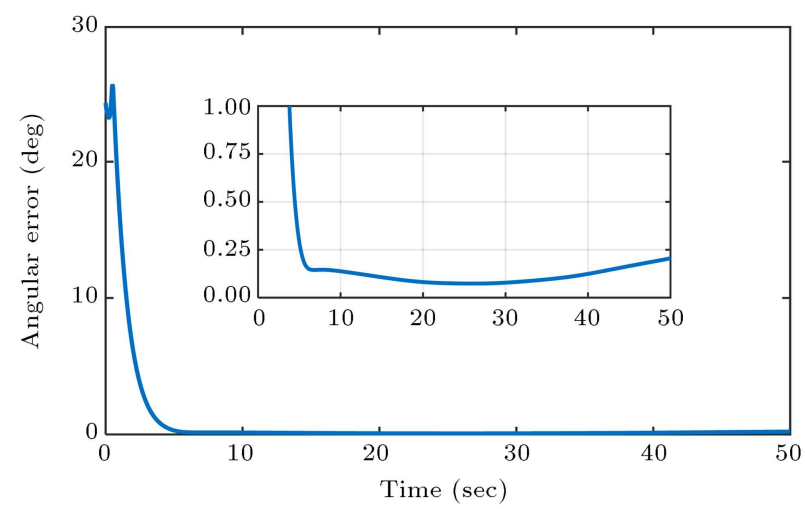

Figure 13. Norm of the end-effector Euler angle errors.

to the equivalent moment of inertia of the end-effector mass in terms of the motor rotation in the mentioned location is defined as follows:

$$
\frac{\mathbf{I}_{\mathbf{m}}}{0.21 m_{b} r^{2}} \text {. }
$$

In the previous simulation, this ratio was $5.6 \%$. In order to further investigate the effect of the inertia of the CPM motors on the tracking error, another 
simulation is performed. The moment of inertia is intentionally considered a large value, $\mathbf{I}_{\mathbf{m}}=0.003 \mathbf{I}_{\mathbf{6}}$, which is 100 times larger than the value in the previous simulation. Therefore, the ratio indicated in Eq. (31) is $560 \%$.

Figures 14 and 15 demonstrate the 2-norm of the translational errors of the two parts in comparison to the previous simulation. In the second simulation, the position error of the end-effector entered the $4 \mathrm{~mm}$ band in $7.6 \mathrm{sec}, 33 \%$ longer than the first simulation. By considering the mass of the end-effector compared with the mobile base, the norm of the mobile base translational errors has not changed significantly. Figure 16 shows that the angular error of the endeffector in the second simulation enters the settling band of $0.4 \mathrm{deg}$ in $4.7 \mathrm{sec}$, while this error for the first simulation enters the band of $0.2 \mathrm{deg}$ in just a little longer time, i.e., 5 sec. Figure 17 demonstrates the mass estimation by the adaptive law. The steadystate estimation of the end-effector mass fluctuates around the nominal value by a small amplitude of $0.1 \mathrm{~kg}$.

Overall, it can be inferred that although the index indicated in Eq. (31) has increased greatly, from $5.6 \%$ to $560 \%$, the tracking errors have not changed significantly.

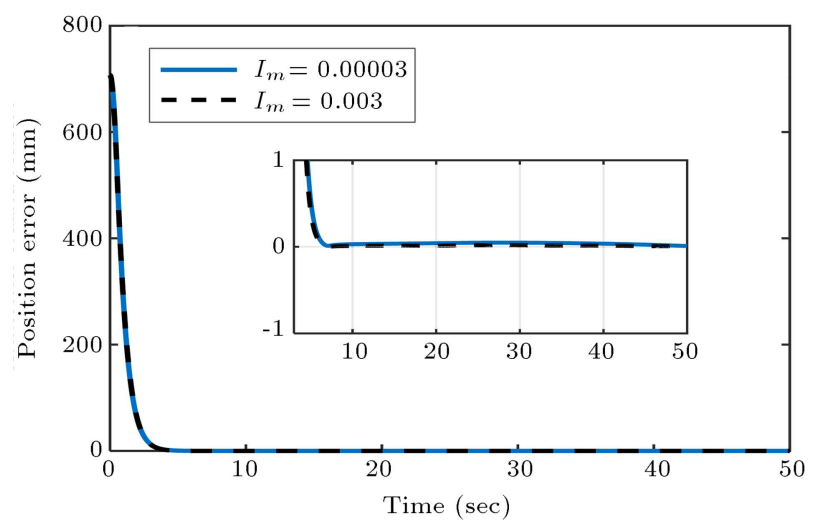

Figure 14. Platform position error.

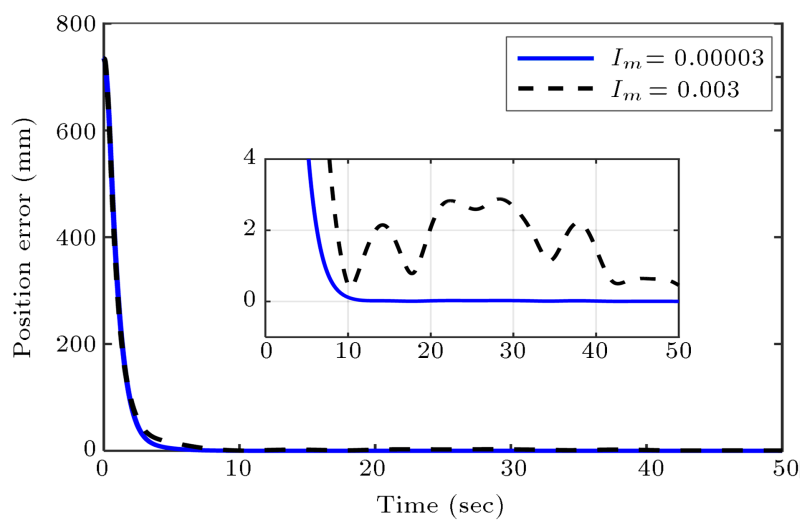

Figure 15. End-effector position error.

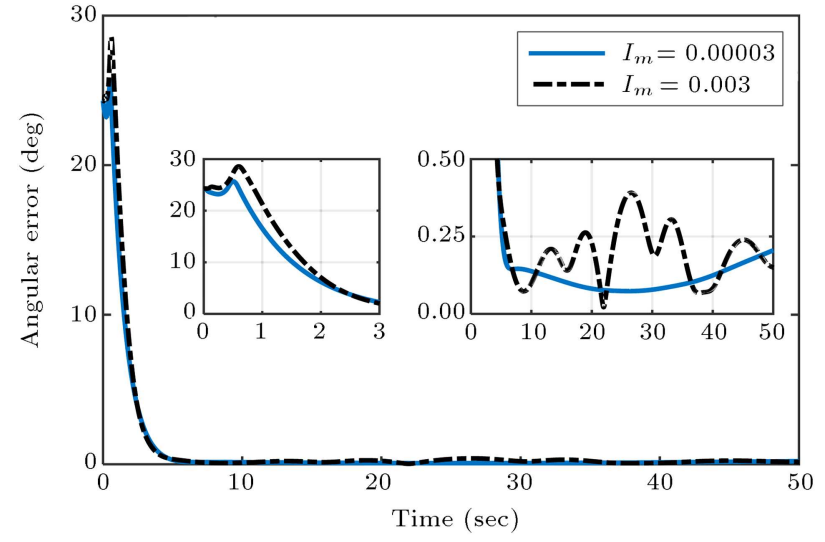

Figure 16. Effect of the moment of inertia of cable motors on the end-effector angular error.

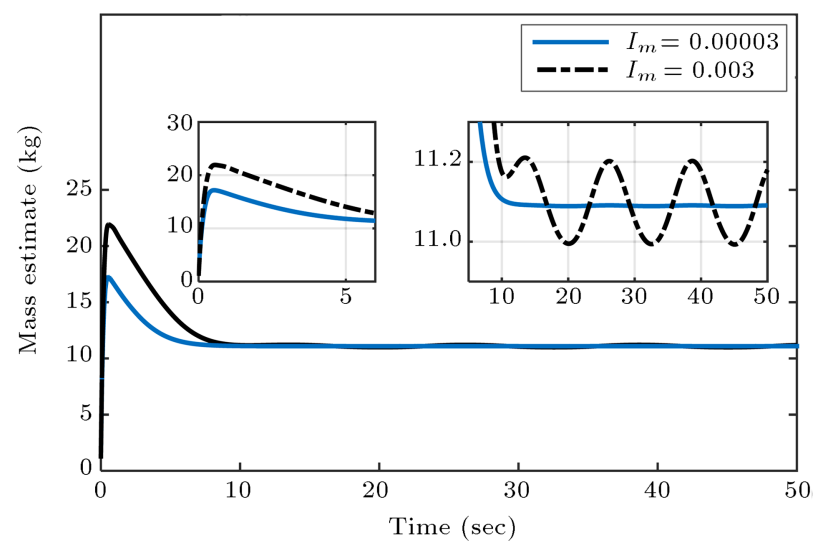

Figure 17. Effect of the moment of inertia of cable motors on the mass estimate.

\section{Experimental results}

The trajectory tracking performance of the controller is evaluated by its implementation on the robotic system, as shown in Figure 18. The CPM part of the robot stands on two independent fixed wheels and one idle caster wheel. The wheels are driven by Permanent Magnet DC (PMDC) motors. The cables of the CPM part of the robot are also driven by PMDC motors. The position feedback of the CPM and mobile base are calculated using the data obtained from the motor encoders. The rotations of the wheels and CPM motors are measured by 600 pulse and 3600 pulse encoders, respectively. Figure 19 shows an interface board, designed for communication between the controller implemented in Matlab and motor drivers and encoders. The communication protocol between the computer and the board is serial. For communicating with the eight sensors and motors of the robot, the board includes eight slave microcontrollers of PIC 18F46K80 type. A similar type of master micro in the board sends a three-bit signal to a multiplexer 4051, which in turn produces an eight-bit signal to activate and deactivate the slave micros. Encoder data are sent to the CCP 


\begin{tabular}{|c|c|c|c|c|}
\hline $\mathbf{x}_{\mathbf{A}}=\left[3.0165 \sin \left(\omega_{A} t+\right.\right.$ & $0.1046)-3.0165 \cos \left(\omega_{A} t+0.1046\right)$ & $\left.\mathbf{0}_{\mathbf{1} \times \mathbf{3}} \quad \omega_{A} t\right]^{T}$, & $\omega_{A}=\frac{2 \pi}{500}$, & \\
\hline $\mathrm{x}_{\mathrm{B}}=\mathrm{x}_{\mathrm{A}}+\left[\begin{array}{c}{ }^{\mathrm{N}} \mathbf{R}_{\mathrm{A}}{ }^{\mathrm{A}} \mathbf{p}_{\mathrm{B}} \\
\mathbf{0}_{\mathbf{3} \times \mathbf{1}}\end{array}\right]$ & & & & \\
\hline${ }^{\mathbf{A}} \mathbf{p}_{\mathbf{B}}=\left[0.05 \sin \left(\omega_{B} t\right)\right.$ & $\left.0.05 \cos \left(\omega_{B} t\right) \quad 0.8-0.1\left|\sin \left(\frac{\omega_{B} t}{4}\right)\right|\right]$ & & $\omega_{B}=\frac{2 \pi}{10}$. & $(32)$ \\
\hline
\end{tabular}

\section{Box II}

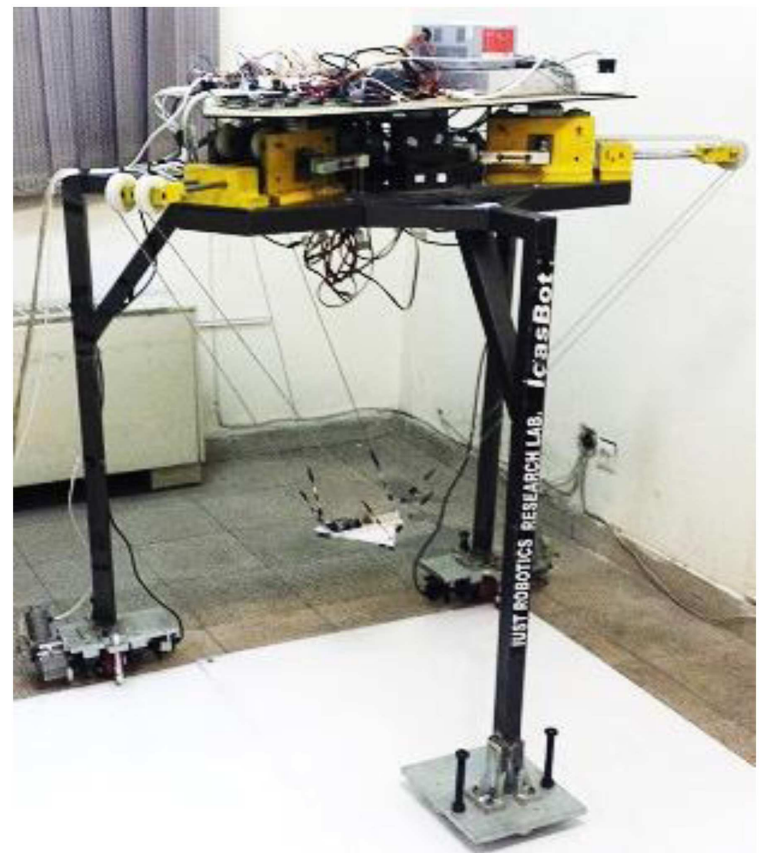

Figure 18. Wheeled mobile Cable-actuated Parallel Manipulator (CPM) of Iran University of Science and Technology.

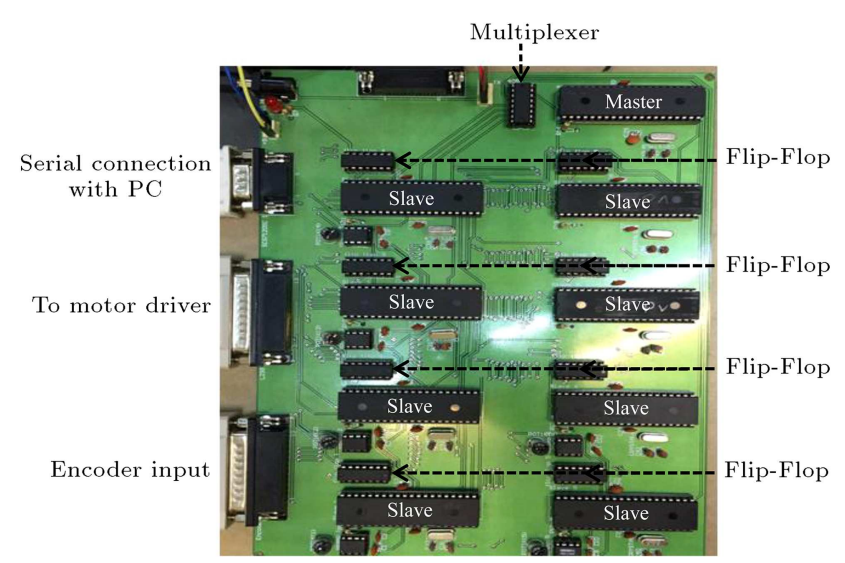

Figure 19. Interface board for transferring the measurement and command data between the computer and robot.

port of the slave micros. The rotation direction of the motors is determined by SN74HC74N flip-flops. The micros are programmed using $\mathrm{C}$ language and MikroC compiler. The PWM signals commanding the motors

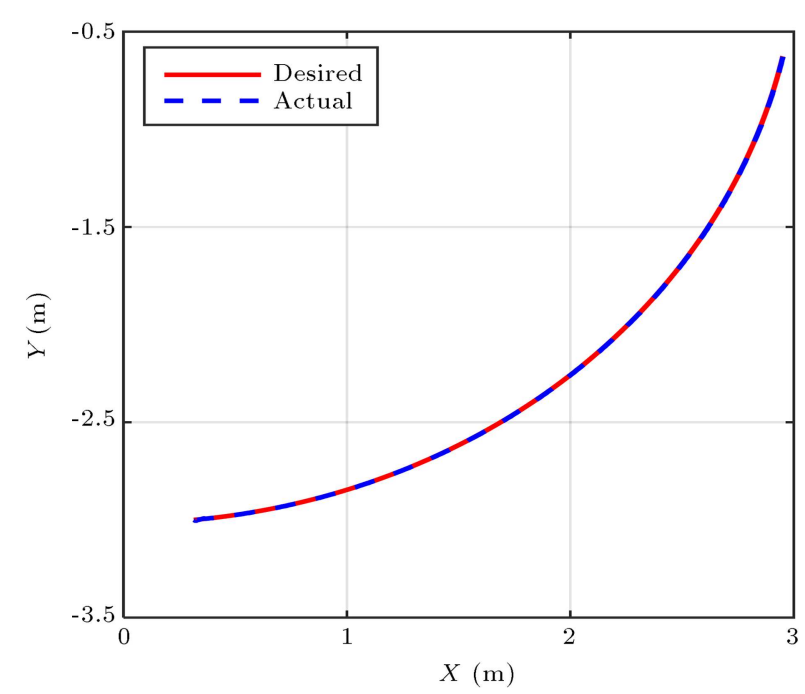

Figure 20. Trajectory tracking of the platform.

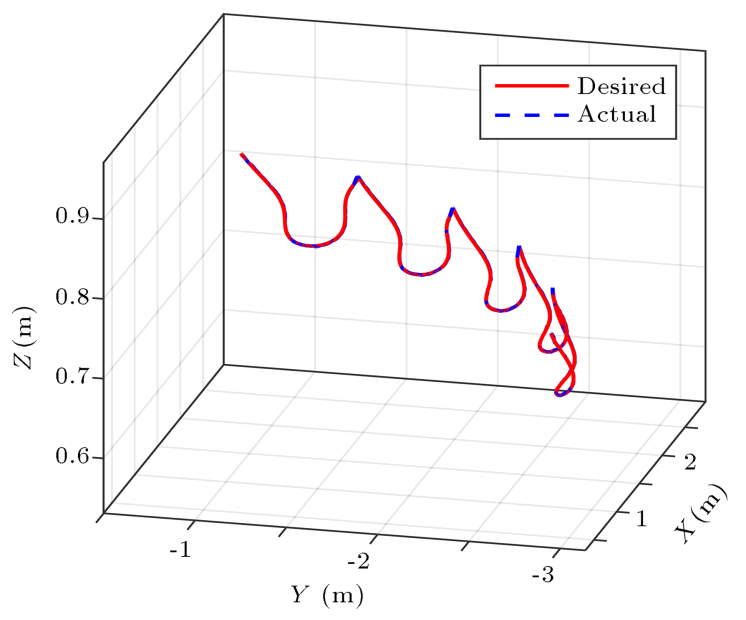

Figure 21. Trajectory tracking of the end-effector.

are obtained by the control input, calculated using Matlab codes.

The test is performed considering an accurate path for the mobile platform and a periodic spiral movement of the end-effector in the mobile base frame (shown in Box II), in which the rotation matrix of the mobile base with respect to the inertial frame is represented by ${ }^{\mathbf{N}} \mathbf{R}_{\mathbf{A}}$. Figures $20-23$ demonstrate that the trajectories of the mobile base and the CPM part 


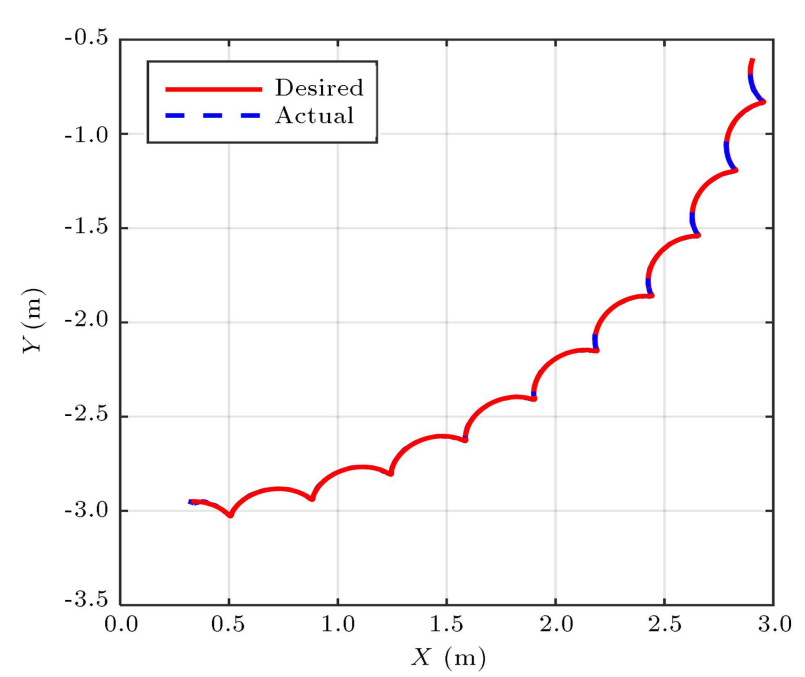

Figure 22. End-effector trajectory in $X Y$ plane.

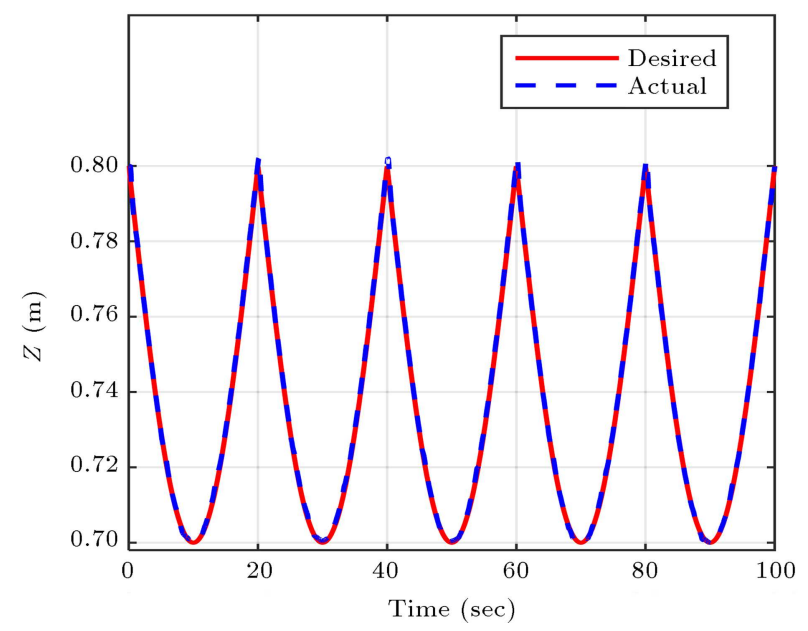

Figure 23. End-effector trajectory in $Z$ direction.

are well in agreement with the desired trajectory. The end-effector initial pose is identical to the start point of the desired trajectory. However, contrary to the initial nonzero desired velocity and acceleration, the system is at rest in the beginning of the tracking. Therefore, Figure 24 demonstrates a $17 \mathrm{~mm}$ overshoot in the end-effector positioning by test and $0.5 \mathrm{~mm}$ overshoot by simulation. Thereafter, the position error of the test quickly decreases to below $5 \mathrm{~mm}$ and remains in this band. Figure 25 shows that the 2-norm of Euler angle errors in the test does not exceed an insignificant value of $1.5 \mathrm{deg}$, except a small $1.69 \mathrm{deg}$ overshoot. The simulation result of the 2-norm of Euler angle errors shows an overshoot of $0.5 \mathrm{deg}$ and, then, the continuous decrease of this value. The end-effector desired orientation, angular velocity, and acceleration in the mobile base frame are zero. However, due to the mobile base angular movement, the desired trajectory of the third Euler angle in the global frame experiences a gentle motion, leading to a small overshoot.

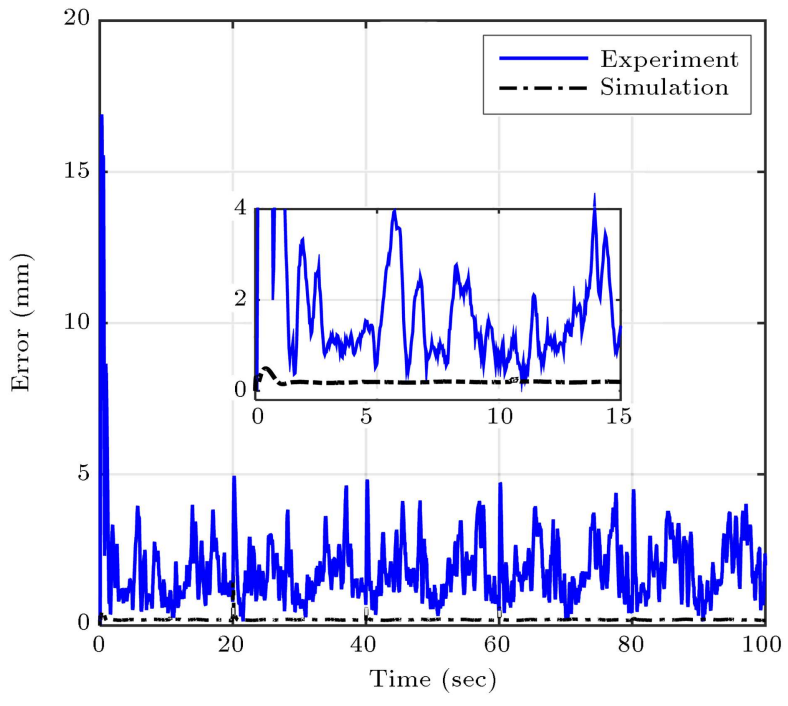

Figure 24. End-effector position error.

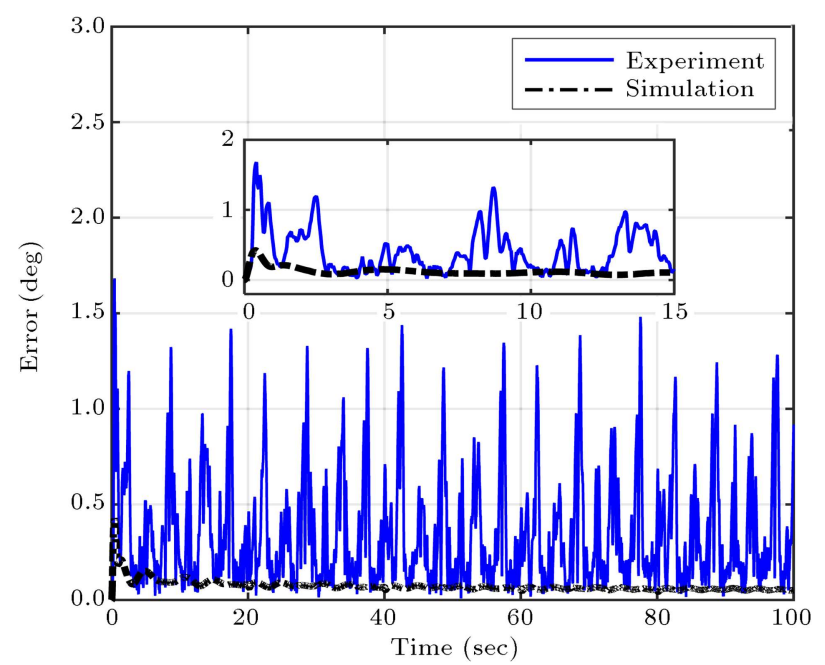

Figure 25. Two-norm of the end-effector Euler angle errors.

The end-effector pose in the global frame is obtained using the mobile base position and the kinematic equations [3]. The position of the mobile base is calculated considering the wheels' rotation. For the experiment, it is assumed that the mobile base undergoes gentle movement maneuver and, consequently, the wheel slip on the ground surface is negligible. Figure 26 demonstrates the wheel path obtained during the test, drawn by black markers next to each wheel as opposed to the desired wheel track in blue lines. As shown in Figure 26, each wheel deviated from the desired path by nearly $7 \mathrm{~mm}$ in the opposite direction. This issue is caused by the flexibility of the legs holding the wheels. In fact, the mentioned deviation results from the legs flexibility, wheels slip, and the performance of the mobile base controller. It is worth noting that since the mobile platform coordinate, employed in the feedback, is nearly between 


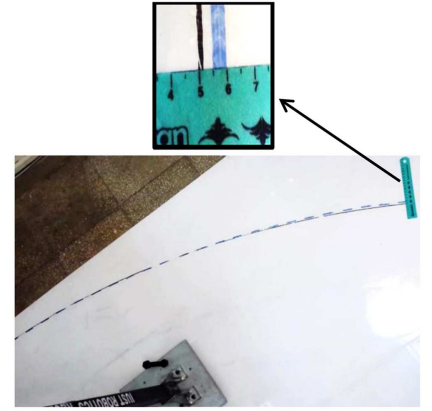

(a)

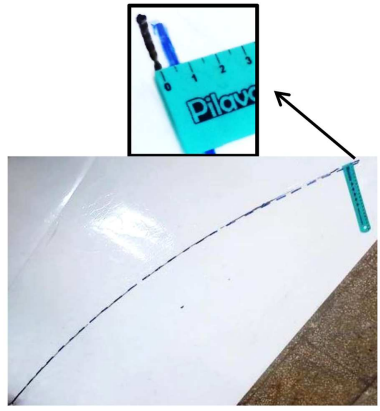

(b)
Figure 26. Comparison of the actual (black solid line) and the desired (blue dashed line) paths of the wheels: (a) Left wheel and (b) right wheel.

the two wheels, the mobile platform position deviation is less than, or at most equal to, the wheels deviation. By strengthening the legs rigidity and also providing the direct measurement of the mobile platform position to include the wheel slip in the feedback, the system error results from only the controller's effectiveness. However, the actual position error of the wheels in this test was only $7 \mathrm{~mm}$ for the $3800 \mathrm{~mm}$ tracking of the mobile base coordinates, i.e., $0.2 \%$, which is a lower value for the mobile platform center.

Figure 27 demonstrates the PWM signals applied to the CPM motors. Due to the presence of friction
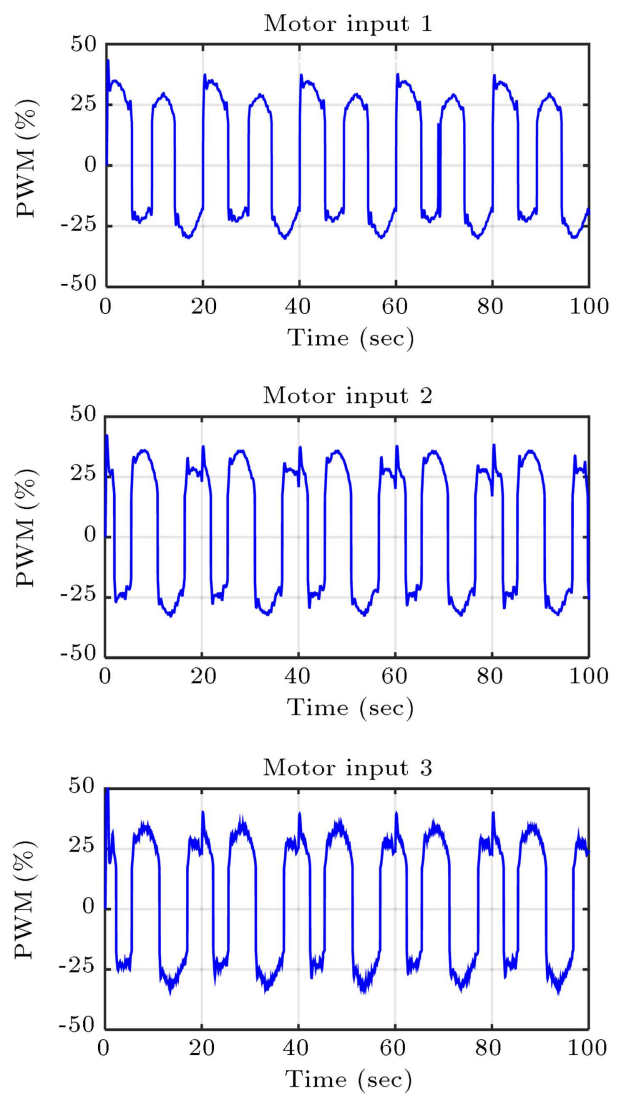

in the motor gearbox, the minimum PWM signals are restricted to $17 \%$. This restriction causes the end-effector to deviate from the desired trajectory when the motors are required to change the rotation direction. The PWM signals applied to the wheel motors are shown in Figure 28. Due to the constant mobile platform velocity, the wheels should rotate at a constant speed. Considering the noticeable mobile platform mass compared with the CPM part, the dynamic coupling between the CPM and mobile base does not affect the computed control input of the wheel motors. Therefore, as shown in Figure 26, the PWM signals applied to these motors remain almost constant during the circular trajectory tracking of the mobile platform. The tracking error is affected by the restrictions of the PWM signals and the control loop delay, caused by the serial connection.

\section{Conclusion}

This study presented the idea of moving an object using a cable-actuated robot. The robot consists of a Cable-actuated Parallel Manipulator (CPM), mounted on a wheeled mobile platform with differential wheels. Kinematic equations of the mobile base and the CPM part of the robot were expressed.

The dynamic equations of motion derived using
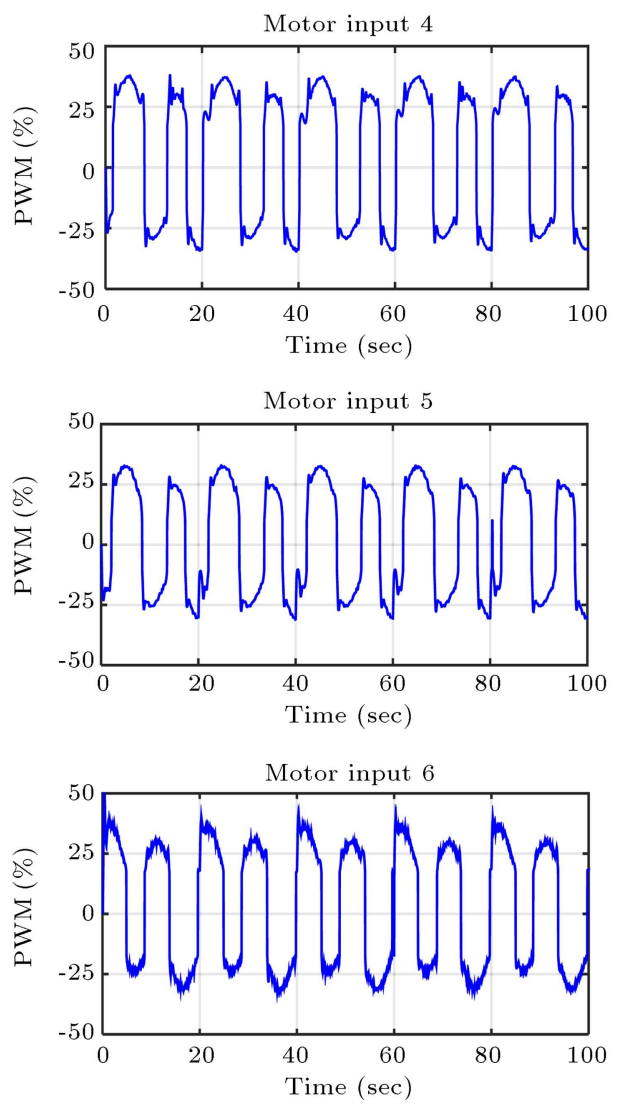

Figure 27. PWM signals applied to the Cable-actuated Parallel Manipulator (CPM) motors. 

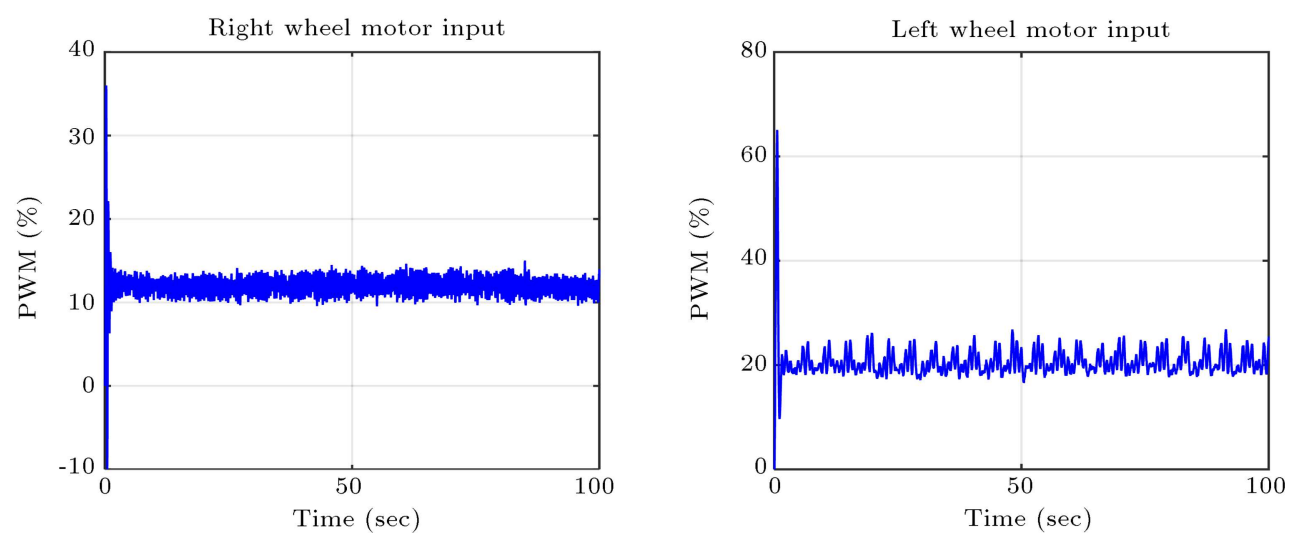

Figure 28. PWM signals applied to the wheel motors.

Euler-Lagrange formulation were presented. The dynamic effect of the moment of inertia of the CPM motors compared with the CPM and the mobile base masses was considered ignorable. Therefore, two different controllers were designed for the CPM part and wheeled platform of the robot. The controllers were designed so as to have a similar error dynamic. For the application of object handling, the inertia specification of the payload is usually unknown. Therefore, an adaptive approach was considered for the end-effector control.

Two case studies were considered to demonstrate the effectiveness of the control approach. They were simulated with the unknown end-effector mass and the control input restrictions. In the first simulation, it was shown that the end-effector orientation, platform position, and the end-effector position well matched the desired trajectory after $5 \mathrm{sec}, 6.4 \mathrm{sec}$, and $11.1 \mathrm{sec}$, respectively. In the second simulation, the tracking accuracy affected by ignoring the moment of inertia of the CPM motors in the control law calculations was studied. Although a large moment of inertia was considered for the CPM motors, it was shown that the tracking errors did not remarkably increase. Further verification of the approach was carried out by an experimental test on a robotic system manufactured in the IUST Lab. It was shown that despite the control loop delay and the restricted PWM interval, the tracking errors were very small, i.e., below $5 \mathrm{~mm}$ for the position error and $1.5 \mathrm{deg}$ for the angular error.

\section{References}

1. Korayem, M.H., Yousefzadeh, M., and Manteghi, S. "Tracking control and vibration reduction of flexible cable-suspended parallel robots using a robust input shaper", Scientia Iranica, 25(1), pp. 230-252 (2017).

2. Oh, S.-R., Ryu, J.-C., and Agrawal, S.K. "Dynamics and control of a helicopter carrying a payload using a cable-suspended robot", Journal of Mechanical Design, 128(5), pp. 1113-1121 (2006).
3. Korayem, M.H., Yousefzadeh, M., and Manteghi, S. "Dynamics and input-output feedback linearization control of a wheeled mobile cable-driven parallel robot", Multibody System Dynamics, 40(1), pp. 55-73 (2017).

4. Korayem, M.H., Yousefzadeh, M., and Susani, S. "Dynamic modeling and feedback linearization control of wheeled mobile cable-driven parallel robot considering cable sag", Arabian Journal of Science and Engineering, 42(11), pp. 4779-4788 (2017).

5. Hu, Y., Zhang, J., Wan, Z., et al. "Design and analysis of a 6-DOF mobile parallel robot with 3 limbs", Journal of Mechanical Science and Technology, 25(12), pp. 3215-3222 (2011).

6. Shao, Z.-F., Tang, X., Wang, L.-P., et al. "Dynamic modeling and wind vibration control of the feed support system in FAST", Nonlinear Dynamics, 67(2), pp. 965-985 (2012).

7. Singh, Y. and Santhakumar, M. "Inverse dynamics and robust sliding mode control of a planar parallel (2-PRP and 1-PPR) robot augmented with a nonlinear disturbance observer", Mechanism and Machine Theory, 92, pp. 29-50 (2015).

8. Asl, R.M., Hagh, Y.Sh., and Palm, R. "Robust control by adaptive non-singular terminal sliding mode", Engineering Applications of Artificial Intelligence, 59, pp. 205-217 (2017).

9. Yang, J., Su, H., Li, Z., et al. "Adaptive control with a fuzzy tuner for cable-based rehabilitation robot", International Journal of Control, Automation and Systems, 14(3), pp. 865-875 (2016).

10. Tuan, L.A., Lee, S.G., Nho, L.C., and Kim, D.H. "Model reference adaptive sliding mode control for three dimensional overhead cranes", International Journal of Precision Engineering and Manufacturing, 14(8), pp. 1329-1338 (2013).

11. Sun, G. and Ma, Zh. "Practical tracking control of linear motor with adaptive fractional order terminal sliding mode control", IEEE/ASME Transactions on Mechatronics, 22(6), pp. 2643-2653 (2017).

12. Roy, S., Roy, S.B., and Kar, I.N. "Adaptiverobust control of Euler-Lagrange systems with linearly 
parametrizable uncertainty bound", IEEE Transactions on Control Systems Technology, 26(5), pp. 18421850 (2017).

13. Babaghasabha, R., Khosravi, M.A., and Taghirad, H.D. "Adaptive robust control of fully constrained cable robots: singular perturbation approach", Nonlinear Dynamics, 85(1), pp. 607-620 (2016).

14. Utkin, V.I. and Poznyak, A.S. "Adaptive sliding mode control", In Aadvances in Sliding Mode Control, pp. 21-53, Springer, Berlin, Heidelberg (2013).

15. Qian, Y., Fang, Y., and Lu, B. "Adaptive robust tracking control for an offshore ship-mounted crane subject to unmatched sea wave disturbances", Mechanical Systems and Signal Processing, 114, pp. 556-570 (2019).

16. Qi, Z., McInroy, J.E., and Jafari, F. "Trajectory tracking with parallel robots using low chattering, fuzzy sliding mode controller", Journal of Intelligent \& Robotic Systems, 48(3), pp. 333-356 (2007).

17. El-Ghazaly, G., Gouttefarde, M., and Creuze, V. "Adaptive terminal sliding mode control of a redundantly-actuated cable-driven parallel manipulator, cogiro", In Cable-Driven Parallel Robots, pp. 179200, Springer International Publishing (2015).

18. Schenk, Ch., Bülthoff, H.H., and Masone, C. "Robust adaptive sliding mode control of a redundant cable driven parallel robot", 19th International IEEE Conference in System Theory, Control and Computing (ICSTCC), pp. 427-434 (2015).

19. Torabi, M., Sharifi, M., and Vossoughi, Gh. "Robust adaptive sliding mode admittance control of exoskeleton rehabilitation robots", Scientia Iranica, 25(5), pp. 2628-2642 (2018).

20. Yamamoto, Y. "Control and coordination of locomotion and manipulation of a wheeled mobile ma- nipulator", PhD Thesis, University of Pennsylvania, Philadelphia, PA (1994).

\section{Biographies}

Moharam Habibnejad Korayem was born in Tehran, Iran in 1961. He received his BS (Hon) and MS degrees in Mechanical Engineering from Amirkabir University of Technology, Tehran, Iran in 1985 and 1987, respectively. He obtained his $\mathrm{PhD}$ degree in Mechanical Engineering from the University of Wollongong, Australia in 1994. He is now a Professor of Mechanical Engineering at Iran University of Science and Technology. His research interests include dynamics of elastic mechanical manipulators, trajectory optimization, symbolic modeling, robotic multimedia software, mobile robots, industrial robotics standard, robot vision, soccer robot, and the analysis of mechanical manipulator with the maximum load-carrying capacity.

Mahdi Yousefzadeh was born in Iran on December 22, 1976. He received his BSc in Mechanical Engineering from Babol Noshirvani University of Technology in 2000 and, then, obtained his MSc from K.N. Toosi University of Technology in 2003 in the field of the automotive system design. He received his $\mathrm{PhD}$ degree in Mechanical Engineering from Iran University of Science and Technology in the field of dynamics, vibration, and control. His research interests include dynamic, control, and vibration analysis of robotic systems. He has 15 years of experience in special machinery design for automotive industry, as well as teaching experience in different universities in Babol, Iran for five years. 